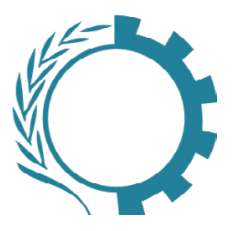

\title{
Vulnerability Analysis of The Tanks of an Oil Refinery to Fire-Induced Dom- ino Effects Based on Graph Theory
}

Mousa Jabbari, Associate Professor, Workplace Health Promotion Research Center; Department of Occupational Health and Safety, School of Public Health and Safety, Shahid Beheshti University of Medical Sciences, Tehran, Iran.

Ahmad Alibabaei, Assistant Professor, Department of E-Learning, Virtual School of Medical Education and Management, Shahid Beheshti University of Medical Sciences, Tehran, Iran.

Amir kavousi, Professor, Department of Epidemiology, School of Public Health and Safety, Shahid Beheshti University of Medical Sciences, Tehran, Iran.

(1) Mehrnoosh Rezvanjah, ( ${ }^{\star}$ Corresponding author), MSc in Industrial Safety, School of Public Health and Safety, Shahid Beheshti University of Medical Sciences, Tehran, Iran. mehrnoosh.rezvanjah@gmail.com

\section{Abstract}

Background and aims: Due to the high amount of flammable materials, the tank farms are vulnerable to fire-induced domino effects. The present study tries to analyze the vulnerability of the crude oil tanks to domino fires, in one of the refineries of Iran. The purpose of vulnerability analysis, is to determine the tanks with the greatest potential to initiate and propagate domino effects, in order to manage the risk of domino fires.

Methods: Graph theory and centrality measures (betweenness and closeness) were used to model domino scenarios and analyze the vulnerability of tanks to domino effects. The ALOHA consequence modeling software was used to model the consequences of the accident scenarios. The R-statistical software and igraph package were used to model the graph and calculate the centrality measures.

Results: The possible domino scenarios in the tanks were modeled as a directed graph, and the tanks were ranked based on the value of betweenness, out-closeness and in-closeness of their respective nodes (which represent the potential for the propagation of domino effects, the potential for initiating domino effects and potential to be affected by other tanks during domino effects, respectively). Also, based on the all-closeness measure, Also, based on all-closeness measure, the most probable sequence of tank involvement in a domino fire was determined.

Conclusion: The use of the graph theory, allows the graphical modeling of domino scenarios and the determination of the most vulnerable tanks in domino scenarios. The results of vulnerability analysis using graph metrics, can be used in the field of domino effects risk management, and the most vulnerable tanks can be prioritized to be assigned to protection measures.

Conflicts of interest: None

Funding: None

\section{Keywords}

Domino effects

Vulnerability analysis

Graph theory

Fire protection

Received: 2019/09/17

Accepted : 2020/07/01 


\section{INTRODUCTION}

Tanks are one of the major hazardous installations in the processing industry, including refineries, which are used to store crude oil and various petroleum products. The presence of multiple tanks containing large volumes of flammable materials that are adjacent to each other, makes the tank farms vulnerable to fireinduced domino effects; therefore, only by analyzing the accidents related to tanks individually, the risk management of tanks will not be sufficient, regardless of the possibility of chain scenarios among tanks. Thus, the present study tries to analyze the vulnerability of the tanks to the fire-induced domino effects in one of the refineries of Iran. The purpose of this vulnerability analysis is to determine and protect the tanks that have the greatest impact on the initiation and propagation of domino scenarios, in order to manage the risk of these chain accidents. In the present study, the graph theory model has been used as one of the new graphical methods to model the domino scenarios between tanks, and also to analyze their vulnerability. Graph measures have so far been used in studies related to the analysis of the vulnerability of the process industries to domino effects caused by terrorist attacks $(1,2)$, as well as accidental failures (3-5).

\section{METHODOLOGY}

In the graph model, the hazardous equipment are considered as graph nodes, and the probability of accident's propagation between equipment is also indicated by the weight of the edges between the nodes. Modeling of the domino scenarios between tanks as a directed graph, makes it possible to analyze the vulnerability of tanks to domino effects, using the graph measures. Using graph measures, the vulnerability indices of tanks (that represent the potential for initiating and propagating the domino effects) can be quantified, and thus tanks can be compared with each other, in terms of their participation in the onset and propagation of the domino events. The steps to model domino scenarios and analyze the vulnerability of hazardous equipment using graph theory are as follows:

1. Identifying the major hazardous equipment as graph nodes: This case study was conducted on 9 tanks containing crude oil. In order to model the domino scenarios in the studied tanks as a graph, 9 nodes corresponding to each tank were considered.

2. Determining the accident scenario for each of the hazardous equipment: According to the type of equipment selected for the study, chemical contents as well as their physical and operational conditions, the accident scenario was considered (both as an initial accident and as a domino-induced accident) for all tanks, in terms of major release and the pool fire due to the immediate ignition.

3. Determining the escalation vectors according to the accident scenario and calculating their severity: Considering the pool fire as the accident scenario related to the tanks, the corresponding escalation vector of the heat radiation was considered. In this study, ALOHA consequence modeling software was used to model the outcome of the pool fire scenario in each of the tanks, and to calculate the intensity of the escalation vectors in the location of other tanks. ALOHA software has been used in various studies to model the consequences of the release of hazardous materials, including toxic material releases and various types of fire $(6,7)$.

4. Comparing the escalation vectors with the threshold value and determining the probable target equipment (determining the graph edges): In order to determine the probable target tanks related to fire in each tank (which can participate in the formation of secondary scenarios and escalation of the primary accident), the values of the escalation vector (here the thermal radiation), were compared with the threshold value $(15 \mathrm{~kW} / \mathrm{m} 2)$; in cases where $\mathrm{Q}_{\mathrm{ij}}$ (the amount of heat radiation received by tank $j$, due to a fire in tank $i$ ) was greater than or equal to the threshold value, tank $j$ was considered the probable target of the fire scenario in tank $i$, and this relationship was modeled by drawing an edge from node $i$ to node $j$. By determining the edges at this stage, the graph structure was formed qualitatively.

5. Calculating the weight of the graph edges: For a meaningful modeling of the domino scenarios in the form of a weighted graph, the weight of each edge was assigned as the ratio of the threshold value to the corresponding escalation vector value of that edge (35 ); thus, if the heat radiation value corresponding to an edge is greater, there is shorter distance between the two nodes connected by that edge, and the effect they have on each other during a domino event is greater as well.

6. Modeling domino scenarios as a graph and calculating nodes' centrality measures (vulnerability indices of the corresponding equipment): $\mathrm{R}$ statistical software and igraph package were used to graphically model domino scenarios in a graph form. $\mathrm{R}$ software is an open-source statistical programming platform in which, the igraph packages can be used to generate graphs and to study their properties. In the previous steps, graph nodes, edges between nodes, as well as the weight assigned to each edge were determined. At this stage, a graph with specified characteristics 
was modeled and plotted. Using the functions in the $\mathrm{R}$ software, the centrality measures (betweenness, incloseness, out-closeness and all-closeness) for each node and consequently, the vulnerability indices of their respective tanks were calculated.

7. Equipment vulnerability analysis based on the graph measures: According to the graph theory, the betweenness index of a node, shows how many graph nodes are in need of this node in order to communicate faster (with fewer intermediaries). The higher the betweenness of a node, the more it is able to facilitate the spread of an effect between other nodes in the graph. From the perspective of the accident propagation between tanks, it is also argued that the tank with the highest betweenness index has the greatest potential for the propagation of domino effects to all tanks. The index of in-closeness of a node in the graph, indicates the speed of access of other nodes to it, and the higher index for a node, indicates that it can be affected by other nodes in a shorter time. The higher in-closeness index of a tank, indicates its high susceptibility to be impacted by all studied tanks during different domino scenarios. The node with the highest amount of out-closeness has a faster access to other nodes, and can affect them in a shorter time. Therefore, it can be argued that starting an accident from a tank with the highest amount of out-closeness, can cause other tanks to engage more rapidly and lead to a more severe domino incident. By definition, the all-closeness index for a node, shows the extent to which that node has access to other nodes and the extent to which they are accessible. Thus, this index can show the capability of a tank to have an impact on other tanks, and also the susceptibility to be affected (damaged) by them during domino effects collectively. It is possible to predict the most probable sequence of the involved tanks in a chain of accidents, by using this index $(3,4)$.

\section{RESULTS}

The magnitude of all escalation vectors were calculated by modeling the defined fire scenario in each tank, using ALOHA software. Table 1, shows the intensity of the escalation vectors related to the fire scenario of each tank at the location of the target tanks. A graph with 9 nodes and 40 edges was modeled using the $\mathrm{R}$ software, and the weight of each edge was assigned. Figure 1, shows the graph drawn

Table 1. Amounts of the thermal radiation $\left(\mathrm{kW} / \mathrm{m}^{2}\right)$ caused by fire in tank $\mathrm{i}$ at the location of the target tank $\mathrm{j}$.

\begin{tabular}{|c|c|c|c|c|c|c|c|c|c|}
\hline $\mathrm{i}$ & 1 & 2 & 3 & 4 & 5 & 6 & 7 & 8 & 9 \\
\hline 1 & - & 20 & - & 50 & - & - & - & - & - \\
\hline 2 & $17 / 5$ & - & 85 & 60 & 40 & 16 & - & - & - \\
\hline 3 & - & 45 & - & 25 & 60 & 69 & 16 & 36 & - \\
\hline 4 & 25 & 34 & 25 & - & 85 & - & - & 16 & - \\
\hline 5 & - & 17 & 34 & 44 & - & 23 & - & 66 & 17 \\
\hline 6 & - & - & 35 & - & 23 & - & 88 & 60 & 42 \\
\hline 7 & - & - & - & - & - & 41 & - & 25 & 61 \\
\hline 8 & - & - & 15 & - & 35 & 35 & 25 & - & 85 \\
\hline 9 & - & - & - & - & - & 16 & 35 & 43 & - \\
\hline
\end{tabular}

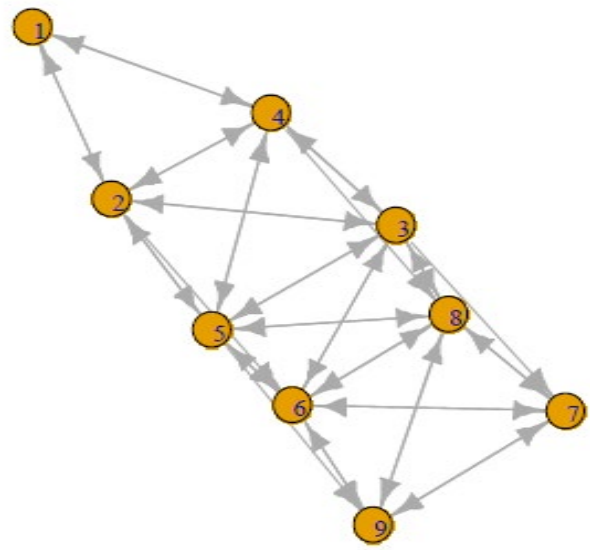

Fig. 1. Graph modeling corresponding to domino scenarios in the studied tanks, using R software. 


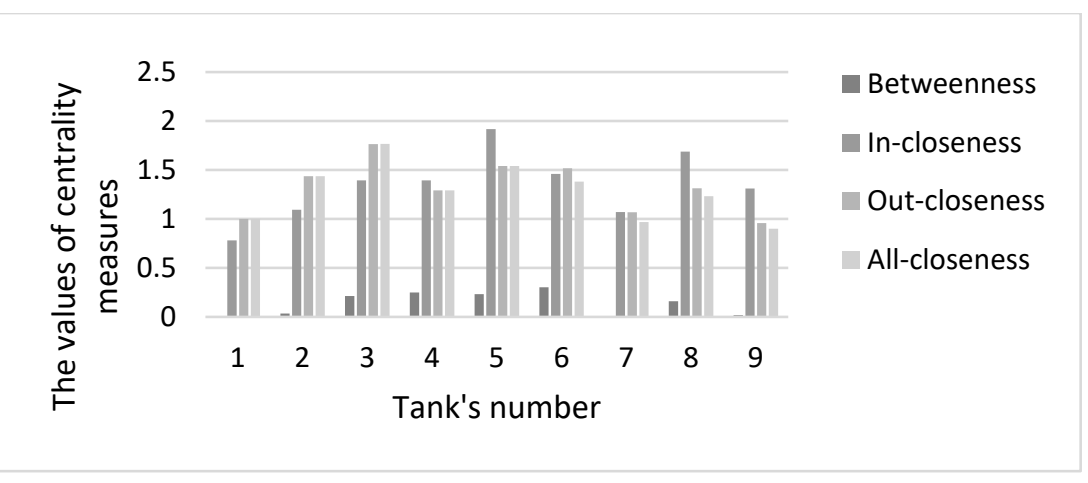

Fig. 2. Comparison of tanks based on the centrality measures.

in the $\mathrm{R}$ software. The target tanks related to the fire scenario in each tank and possible domino scenarios, are shown in the plotted graph. The values of the centrality measures for the graph nodes (tanks) were calculated using the functions in the $\mathrm{R}$ software. As figure 2 shows, tanks 6, 4, 5, 3, 8, 2, 9, 1 and 7 have the highest values of the betweenness index (susceptibility to propagation of domino effects), respectively. Tanks $3,5,6,2,8,4,7,1$, and 9 have the highest values of the out-closeness index (susceptibility to trigger severe domino effects), respectively. Tanks 5, 8, 6, 3, 4, 9, 2, 7 and 1 have the highest values of the in-closeness (susceptibility to be impacted during different domino scenarios), respectively. Tanks $3,5,2,6,4,8$, 1,7 and 9 have the highest values of the all-closeness index (potential to trigger severe domino effects and to be impacted during different domino scenarios collectively), respectively; thus this arrangement is the most probable sequential involvement of tanks in chain fires.

\section{DISCUSSION}

Vulnerability analysis using the graph measures, not only makes it possible to identify critical tanks initiating and propagating domino effects, but also by using the results, helps in the initiation of plans for the safety management of these accidents. Just as the removal of the node with the highest betweenness index from the graph, leads to the greatest disconnection in the graph structure; the protection of the tanks with the highest betweenness index, also leads to the interruption of critical paths of accident propagation between the tanks. Protecting the tanks has the greatest impact on other tanks (the tanks with the highest out-closeness index), and also prevents the onset of the most severe domino scenarios. In studies conducted so far, various approaches have been used to manage the risk of domino effects. A study by Khakzad et al. (2017) on the optimal allocation of protection strategies against domino effects, showed that active protection of critical equipment that initiate domino effects, and the passive protection of critical equipment that propagate domino scenarios, is the most effective way to reduce the vulnerability of the chemical plants to domino fire accidents (5). Therefore, the effect of fireproofing (as a passive protection method) four tanks with the highest potential for accident propagation (tanks 6, 4, 5, and 3 , respectively), and the use of sprinkler system (as an active protection method) for the four tanks with the highest potential to trigger domino effects (tanks 3 , 5,6 , and 2, respectively) on the vulnerability of crude oil tanks' layout, has been investigated. To quantify the vulnerability of tanks' layout subject to domino effects, the graph out-closeness score (average of the out-closeness scores of nodes) was used. In the case where there is no protection, the value of graph outcloseness is 1.32, as Table 2 shows, the protection of

Table 2. The effect of the protection measures on the vulnerability of tanks' layout (out-closeness score of the graph).

\begin{tabular}{|c|c|c|c|}
\hline & & Protected tanks & Out-closeness score of the graph \\
\hline \multirow{8}{*}{$\begin{array}{l}\text { The type of } \\
\text { protection measure }\end{array}$} & \multirow{4}{*}{ Fireproofing } & Tank6 & 0.65 \\
\hline & & Tank6 and Tank 4 & 0.39 \\
\hline & & Tank6, Tank4 and Tank5 & 0.28 \\
\hline & & Tank6, Tank4, Tank5 and Tank3 & 0.18 \\
\hline & \multirow{4}{*}{ Sprinkler system } & Tank3 & 1.24 \\
\hline & & Tank3 and Tank5 & 1.03 \\
\hline & & Tank3, Tank5 and Tank6 & 0.93 \\
\hline & & Tank3, Tank5, Tank6 and Tank2 & 0.91 \\
\hline
\end{tabular}


the most vulnerable tanks, especially fireproofing the tanks which contributes to the propagation of domino effects the most, can cause a significant reduction in the vulnerability of the tanks' layout to domino fires.

\section{CONCLUSION}

The graph theory model, is an effective approach for graphical modeling of domino scenarios, and determining the most vulnerable tanks in terms of their potential in initiating and propagating domino effects. The non-probabilistic nature of the graph model, reduces the size of the calculations for vulnerability analysis and the duration required to do so, compared to the probabilistic methods. The results of the vulnerability analysis using the graph metrics, can be used in the field of domino effects risk management, and the most vulnerable tanks can be prioritized to assign protection measures such as, active and passive safety barriers. According to the study, the protection of the most vulnerable tanks (by fireproofing and using the sprinkler system), can reduce vulnerability of the tanks' layout to domino effects. However, the cost of implementing protection measures is also important in choosing the type of protection measure and the number of selected tanks to allocate protection measures (prioritized based on the vulnerability indexes). Given that the protection measures usually require economic justification due to limited financial resources, the vulnerability index of tanks' layout (closeness score of graph) can be used in conjunction with other decision-making criteria, for selecting effective protection strategies (such as cost of implementation) and achieving the optimal economic solutions.

\section{ACKNOWLEDGMENT}

The authors would like to thank the Shahid Beheshti University of Medical Sciences for financial support of the research, and to thank the safety experts and the HSE officials of the Oil Refining Company for their cooperation in the conduction of this research.

\section{CONFLICT OF INTEREST}

The authors declare that there are no conflict of interest regarding the publication of this manuscript.

How to cite this article:

Mousa Jabbari, Ahmad Alibabaei, Amir kavousi, Mehrnoosh Rezvanjah. Vulnerability Analysis of The Tanks of an Oil Refinery to Fire-Induced Domino Effects Based on Graph Theory. Iran Occupational Health. 2021 (01 July);18:14.

*This work is published under CC BY-NC 4.0 licence 


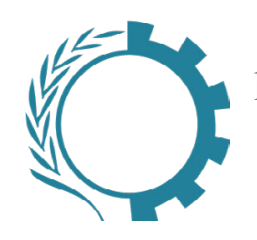

\section{مقاله يُخوهشى}

http://ioh.iums.ac.ir

\section{تحليل آسيبذيذيرى مخازن يكى از بالايشَاههاى نفت نسبت به حوادث زنجير إى

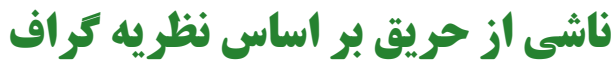

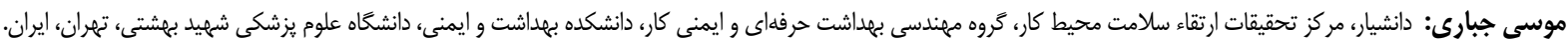

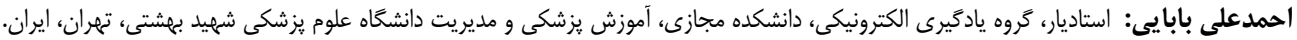

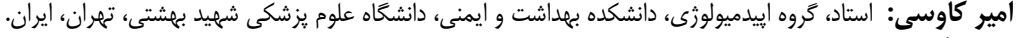

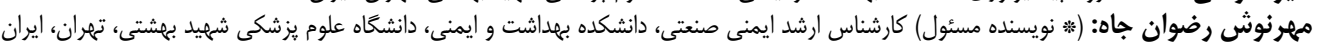
mehrnoosh.rezvanjah@gmail.com

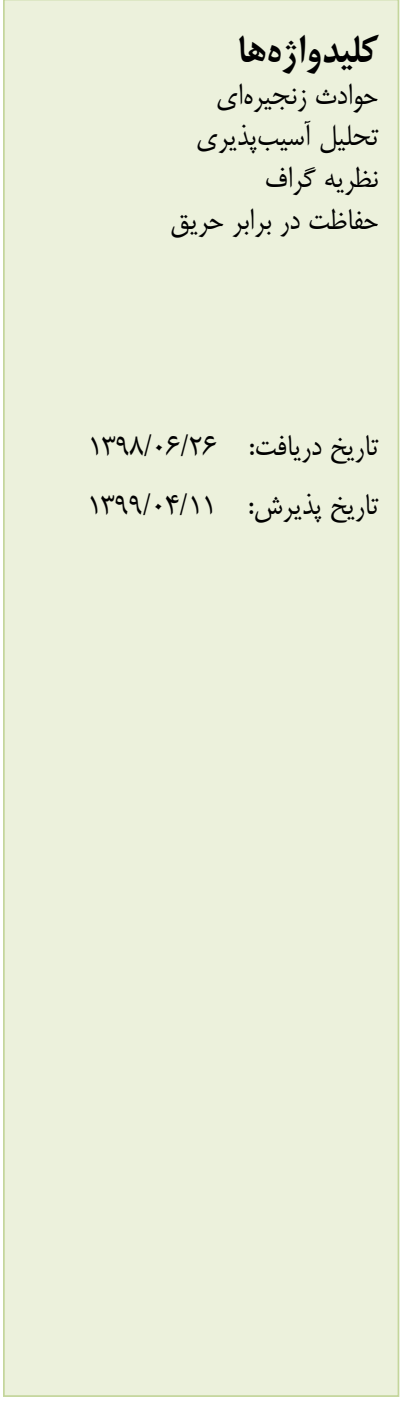

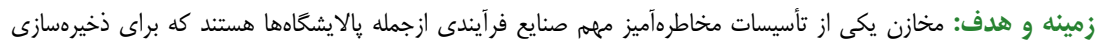

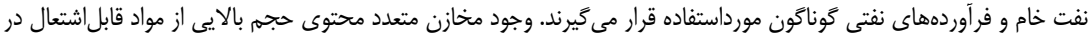

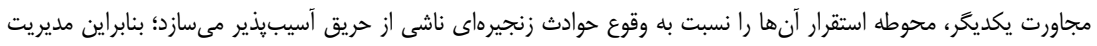

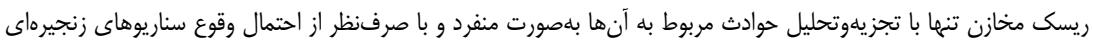

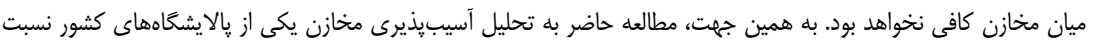

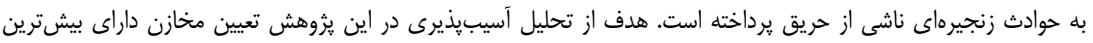

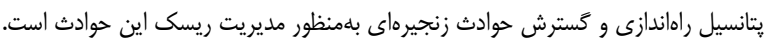

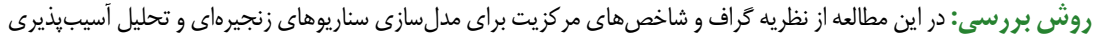

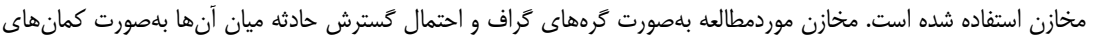

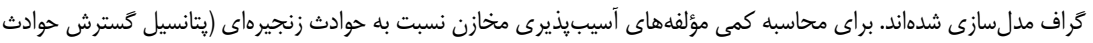

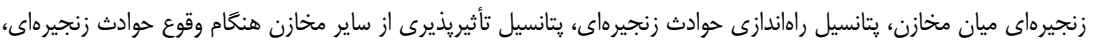

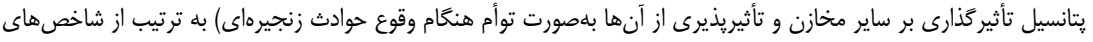

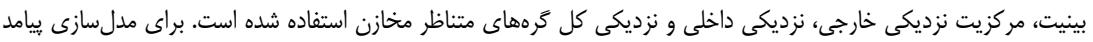

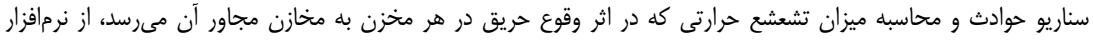

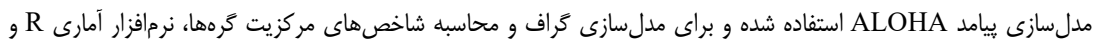

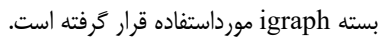

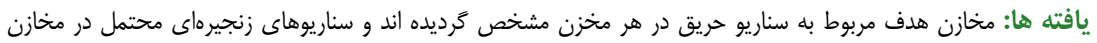

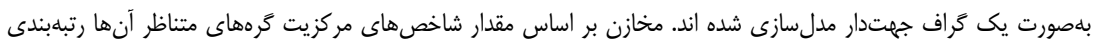

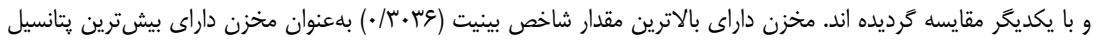

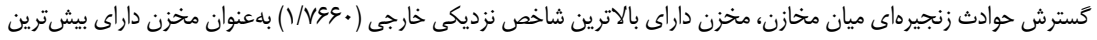

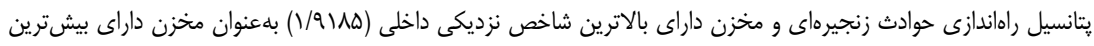

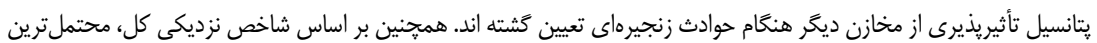

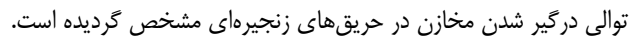

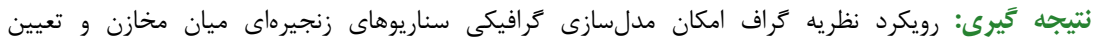

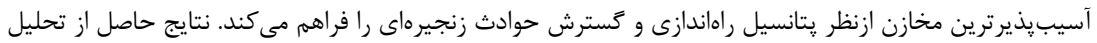

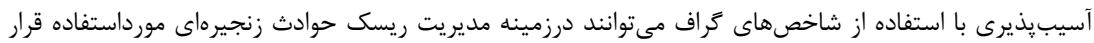

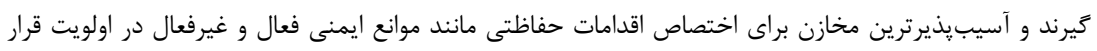

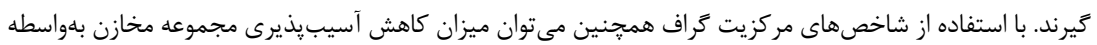

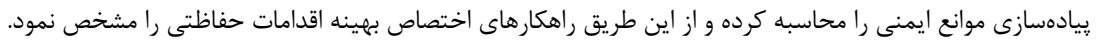

$$
\text { تعارض منافع: كزارش نشاده است. }
$$

شيوه استناد به اين مقاله: Mousa Jabbari, Ahmad Alibabaei, Amir kavousi, Mehrnoosh Rezvanjah. Vulnerability Analysis of The Tanks of an Oil Refinery to Fire-Induced Domino Effects Based on Graph Theory. Iran Occupational Health. 2021 (01 July);18:14. 
قراركرفتهاند؛ زيرا وجودِ مخازن محتوى حجم بالايى از

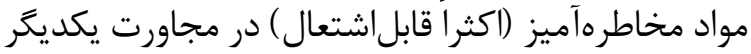

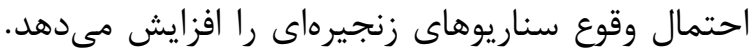

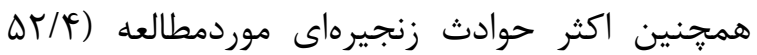

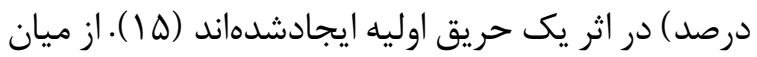

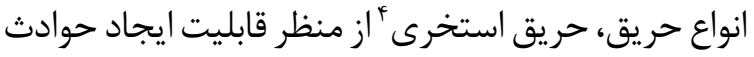

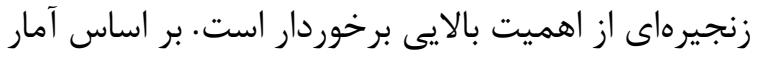

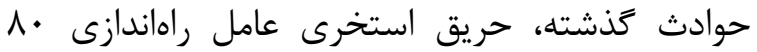

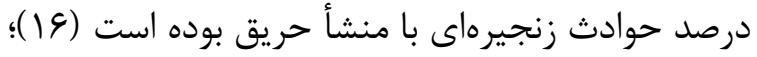

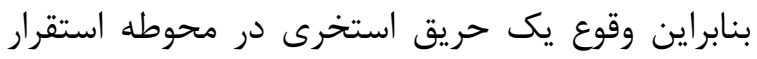

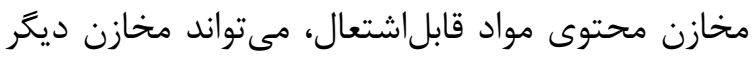

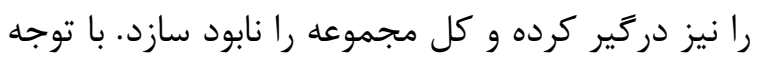

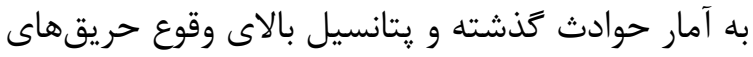

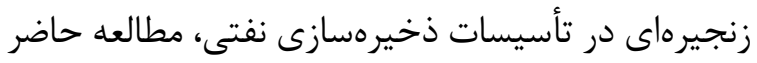

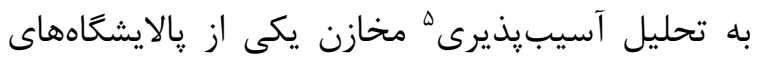

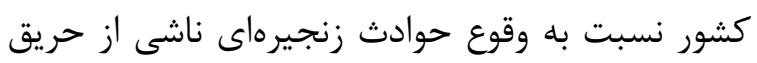

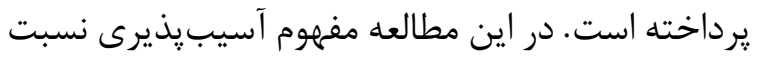

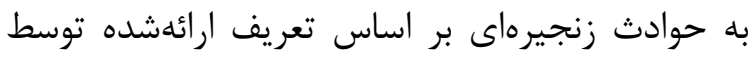

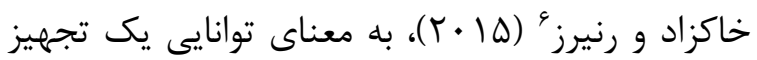

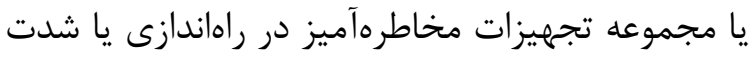

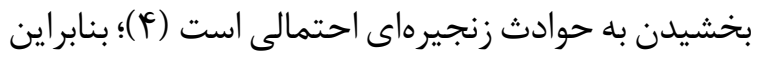

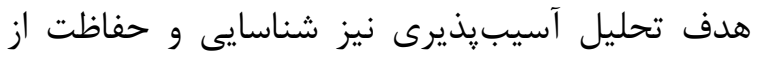

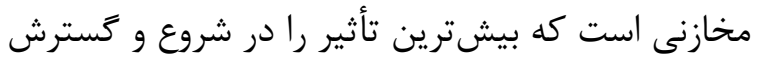

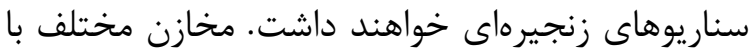

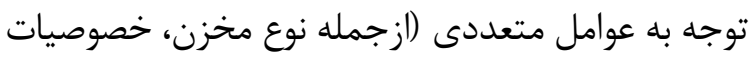

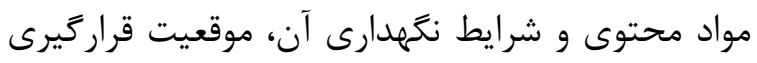

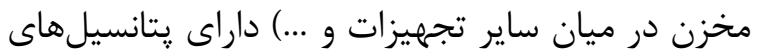

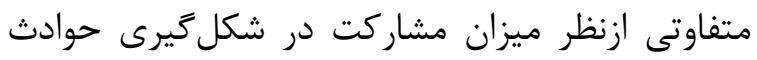

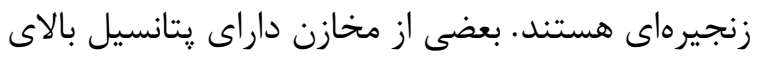

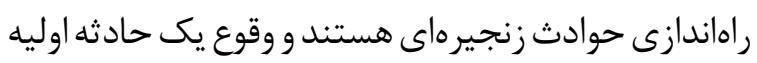

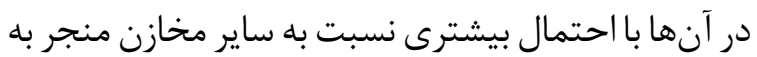

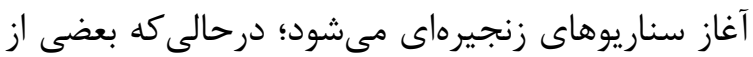

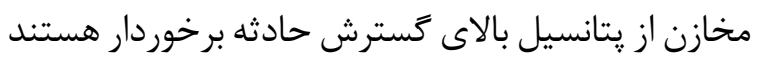

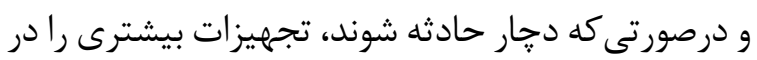

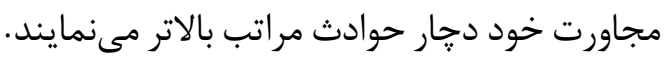

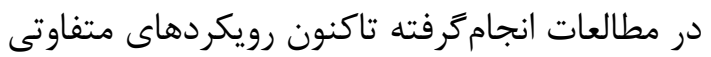

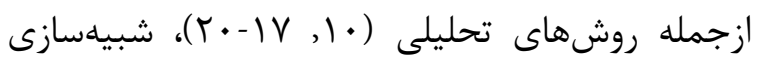

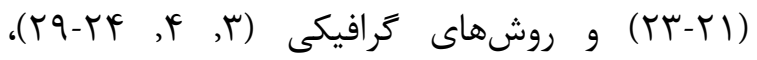

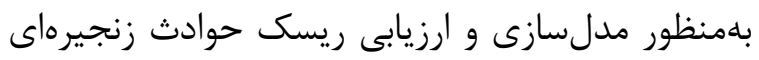

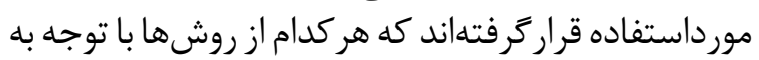

4 Pool Fire

5 Vulnerability

6 Reniers
مقامله

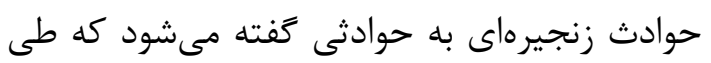

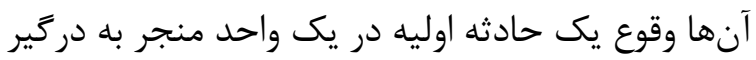

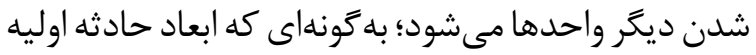

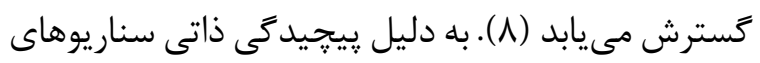

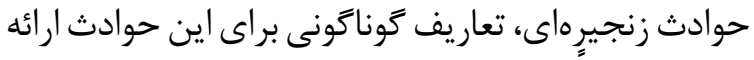

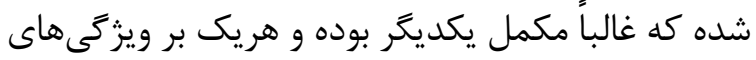

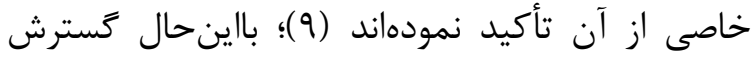

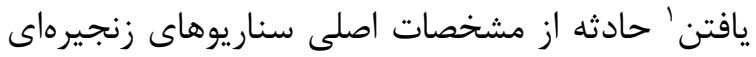

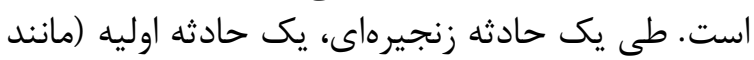

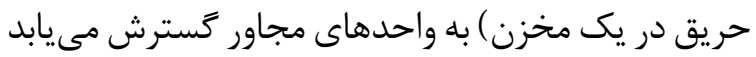

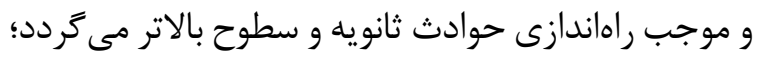

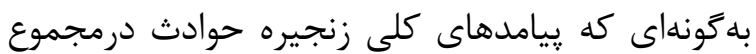

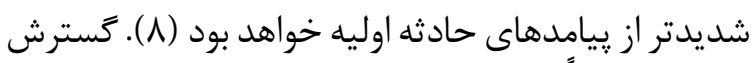

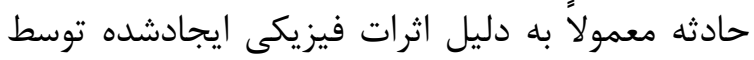

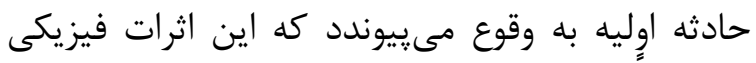

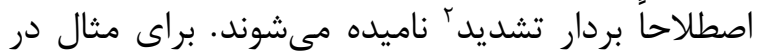

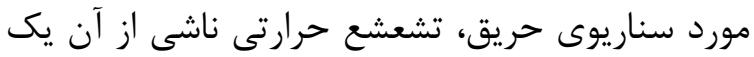

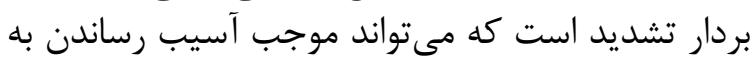

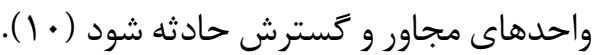

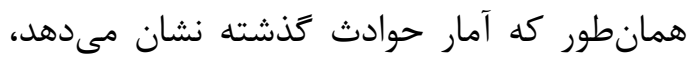

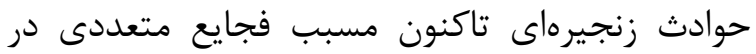

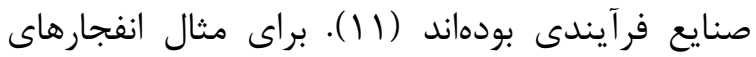

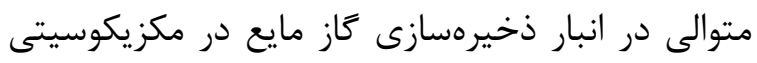

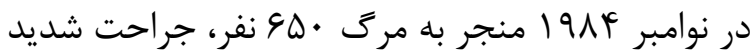

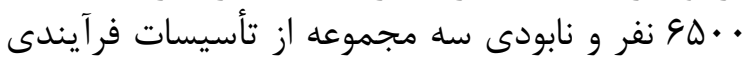

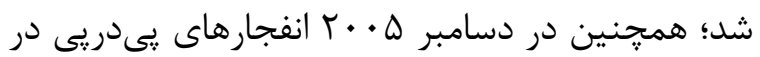

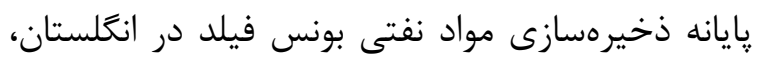

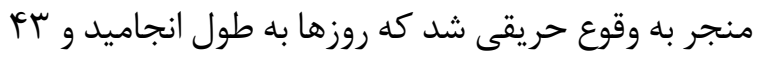

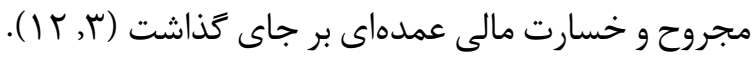

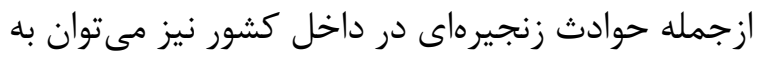

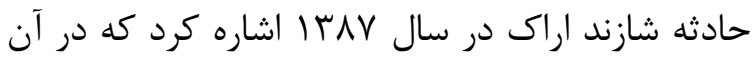

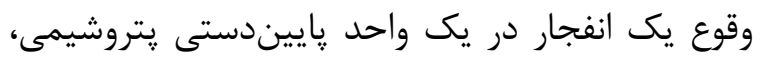

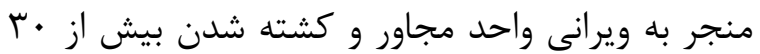

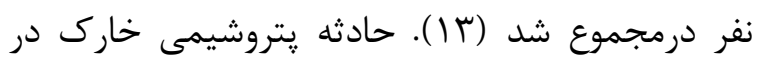

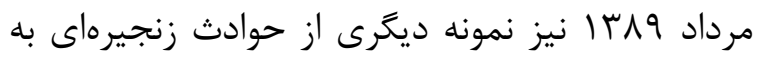

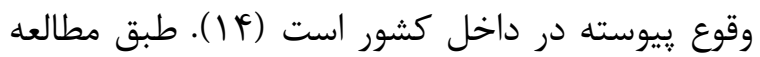

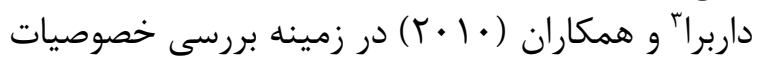

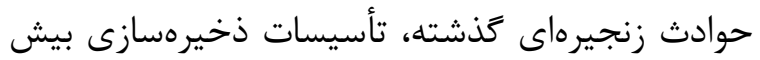

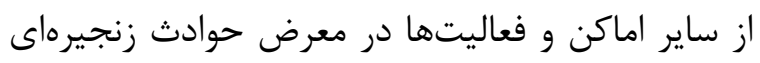

\footnotetext{
1 Propagation

2 Escalation Vector

3 Darbra
}

Iran Occupational Health. 2021 (01 July);18: 14 
استفاده از شاخصهاى گراف انجام گرفت. در اين مطالعه

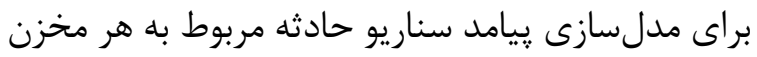

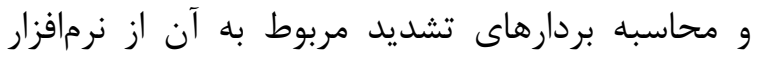

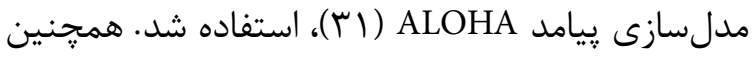

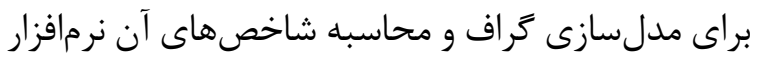

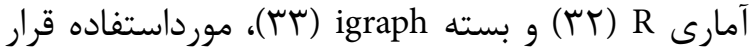

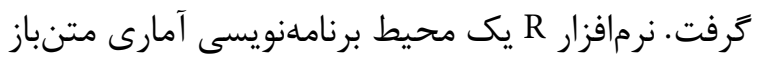

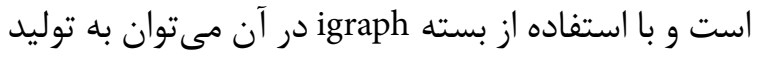

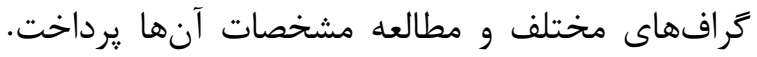

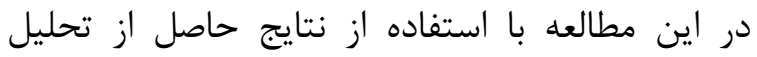

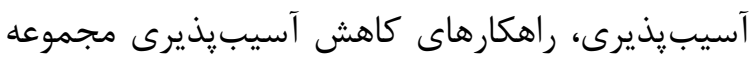

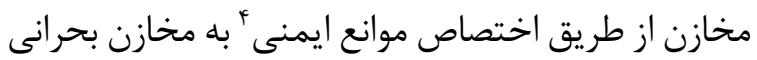

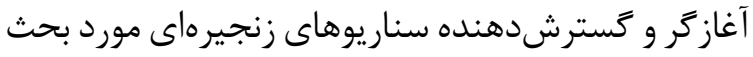

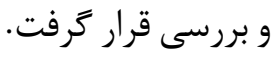

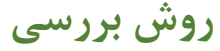

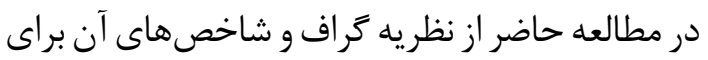

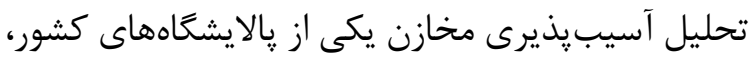

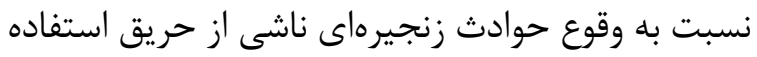

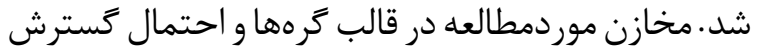

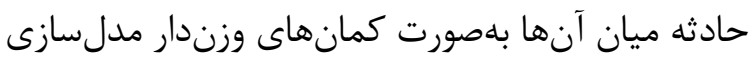

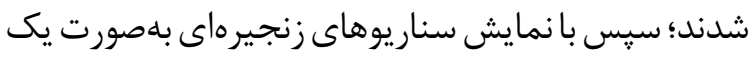

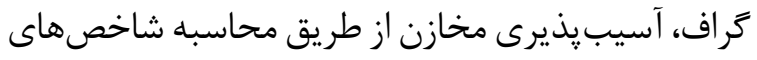

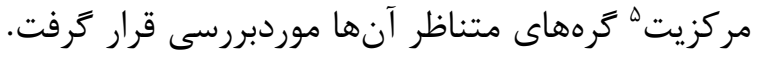

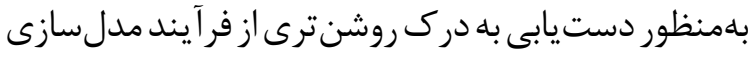

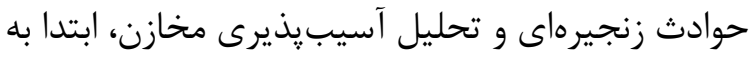

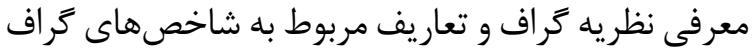

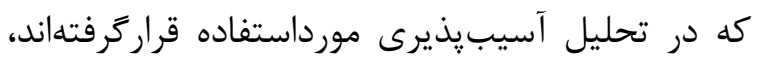

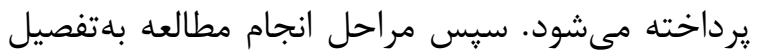

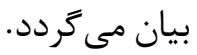

نظريه تراف

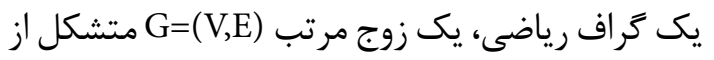

مجموعهاى از كرها كمان ها

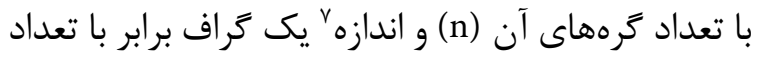

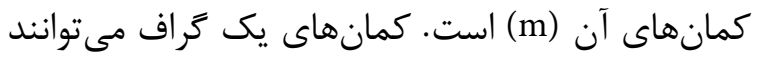

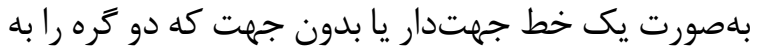

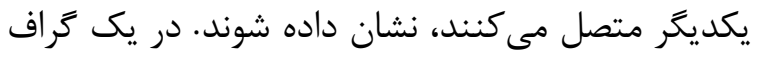

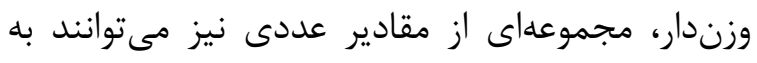

4 Safety Barriers

5 Centrality Measures

6 Order

7 Size
هدف مطالعه و محدوديت زمان اجراى آن از مزاياو معايب

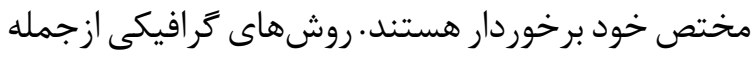

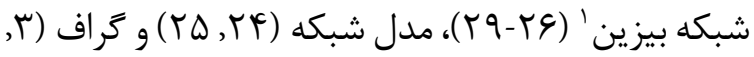

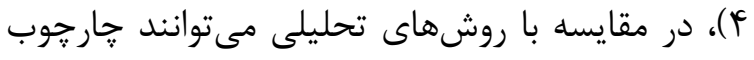

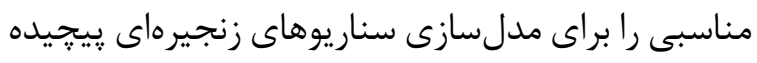

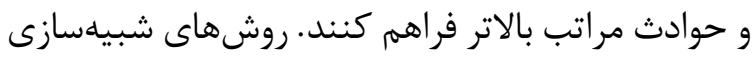

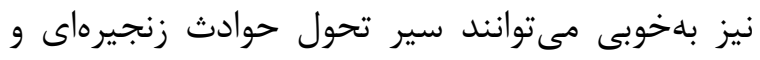

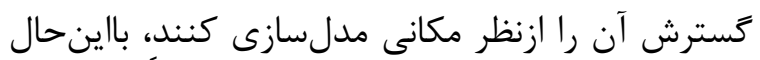

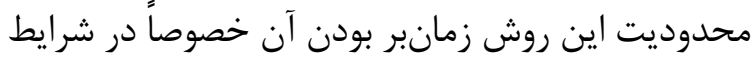

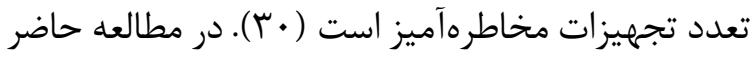

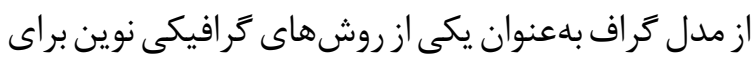

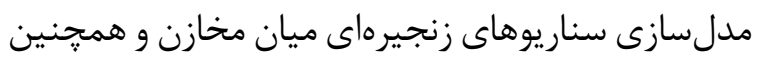
تحليل آسيبيذيرى آنها استفاده شدهاست.

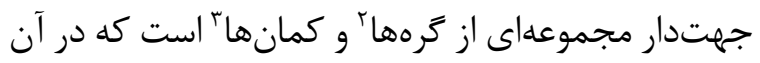

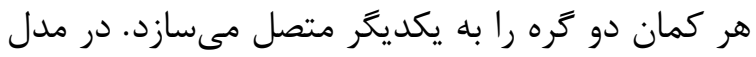

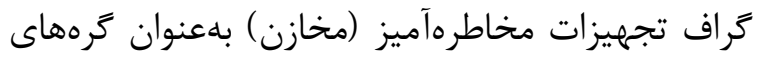

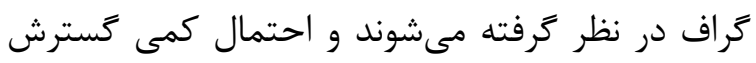

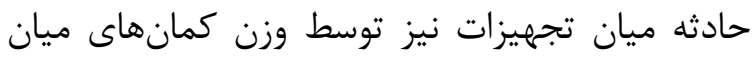

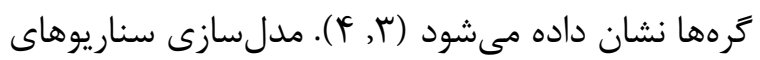

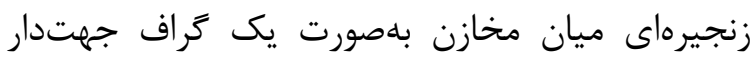

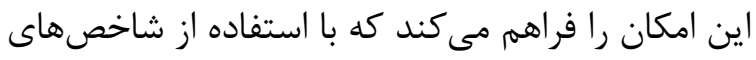

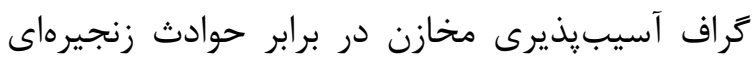

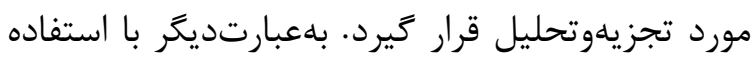

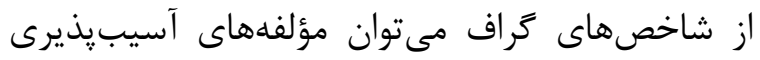

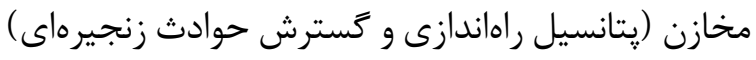

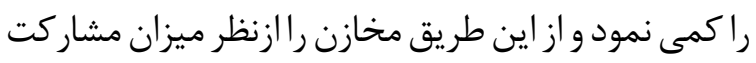

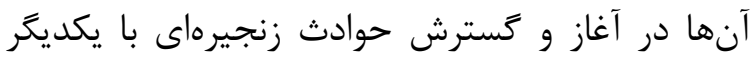

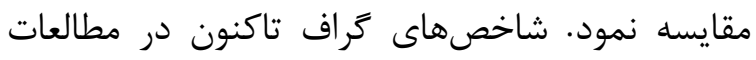

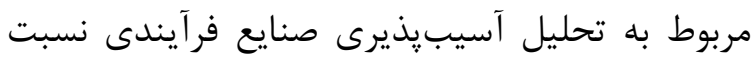

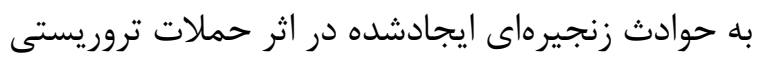

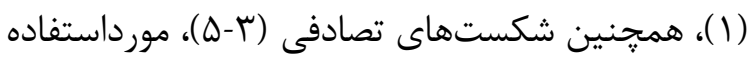

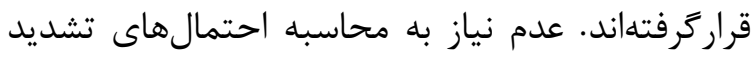

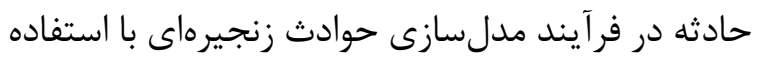

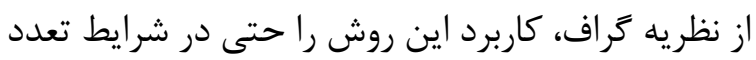

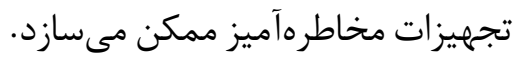

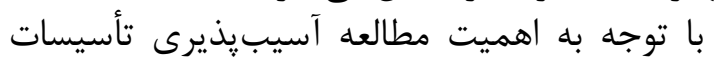

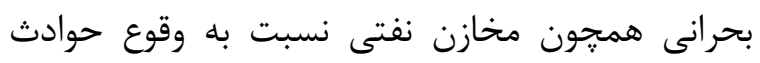

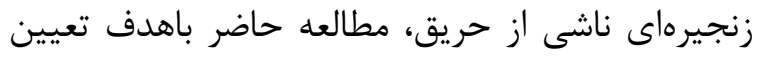

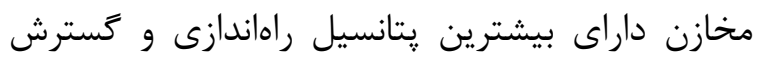

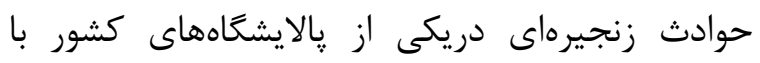

1 Bayesian Network

2 Nodes

3 Edges 
است و با نماد Cماي C نزديكى كل كه با نماد (v-all

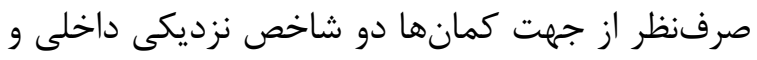

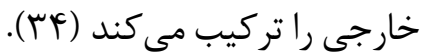

$$
\begin{aligned}
& \text { Cc }- \text { out }(\text { vi })=\frac{1}{\sum_{j} \text { dij }} \\
& \text { Cc }- \text { in }(\text { vi })=\frac{1}{\sum_{j} \text { dji }}
\end{aligned}
$$

كَفتنى است كه بر اساس مقادير مركزيت نزديكى

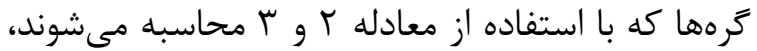

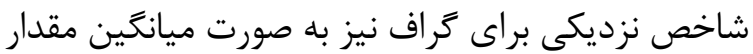

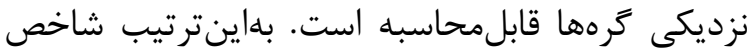
نزديكى خارجى براى كراف كراف (C-out

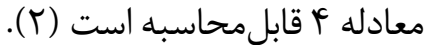

$\mathrm{Cc}-\operatorname{out}(\mathrm{G})=\frac{\sum_{\mathrm{i}=1}^{\mathrm{n}} \mathrm{Cc}-\operatorname{out}(\mathrm{vi})}{\mathrm{n}}$

$$
\text { فرآيند مدل سازى حوادث زنجيراى بهصورت تراف و }
$$
تحليل آسيب بذيرى تجهيزات مخاطرهآديز

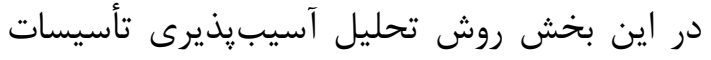

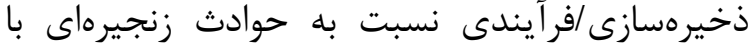

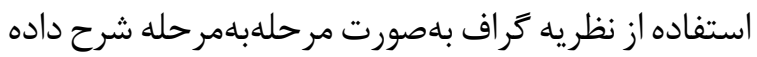

$$
\begin{aligned}
& \text { مرحله اول: تعيين تجهيزات مخاطرةآميز مهمر بهعنوان } \\
& \text { كر مهاى كراف }
\end{aligned}
$$

كام اول مدل سازى حوادث زنجيرهاف نظريه گراف، تعيين تجهيزات مخاطرهآميز مهارم (تجهيزات

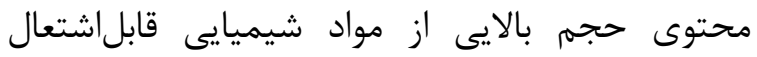

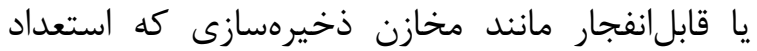

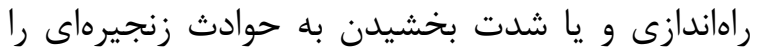

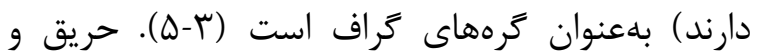

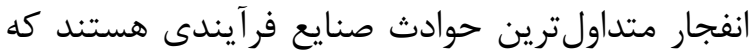

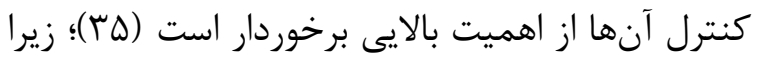

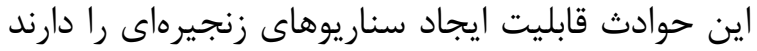

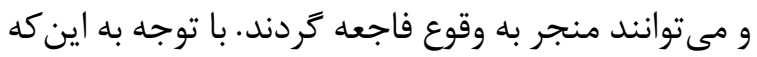

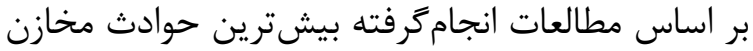

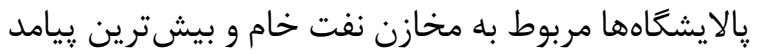

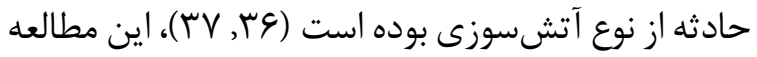

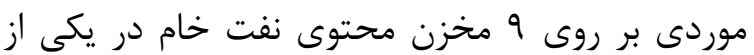

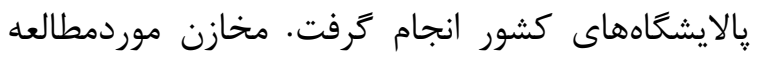

هريك از ₹رهها يا كمانهاى گر اف نسبت داده شوند.در اين

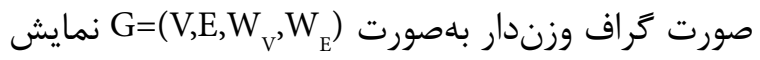

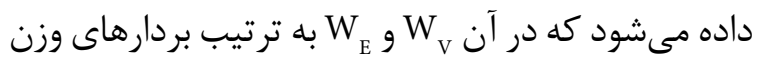

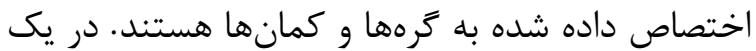

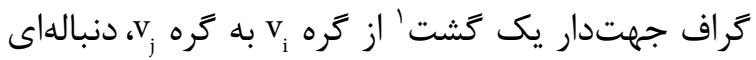

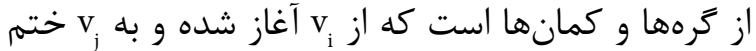

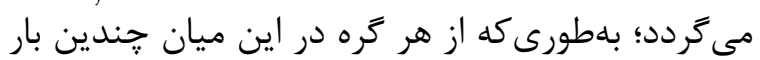

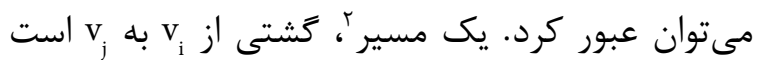

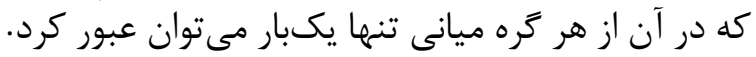

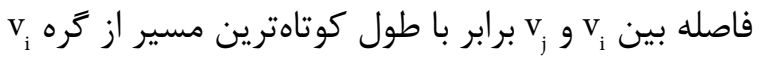

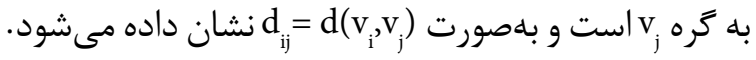

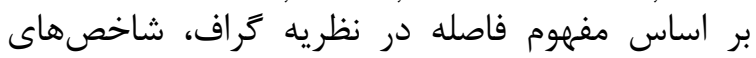

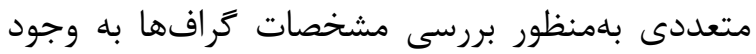

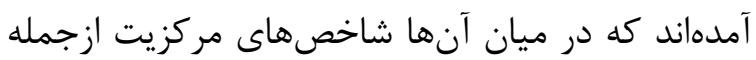

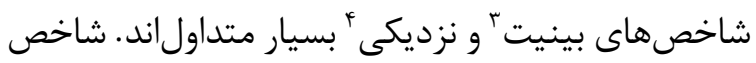

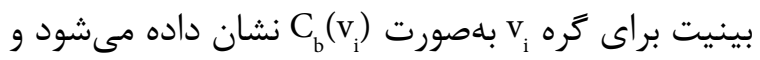

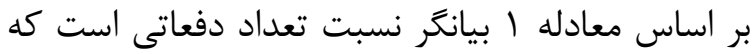

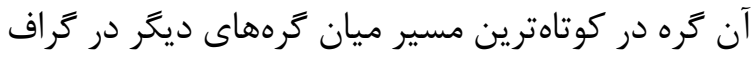
قرار مى كيرد. در اين معادله

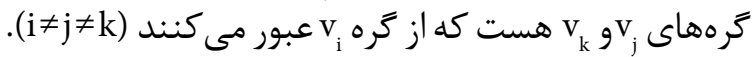

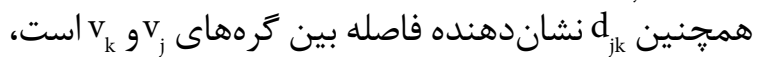

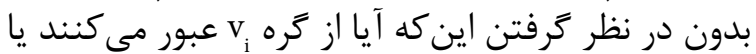

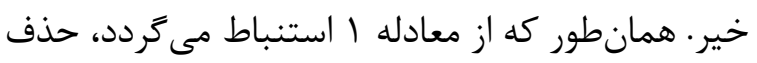

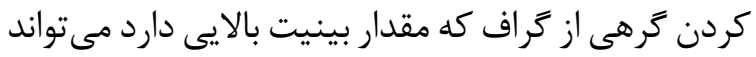

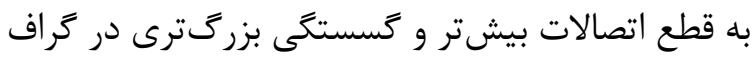

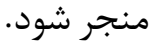

$\mathrm{Cb}(\mathrm{vi})=\sum_{\mathrm{j}, \mathrm{k}} \frac{\mathrm{djk}(\mathrm{vi})}{\mathrm{djk}}$

شاخص متداول ديخر در زمينه بررسى مشخصات

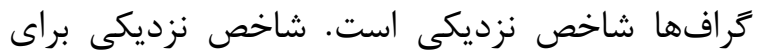

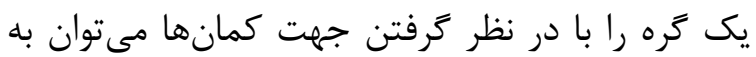

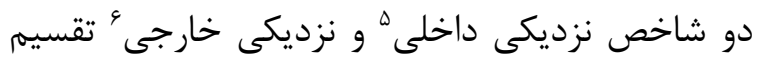

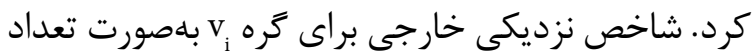

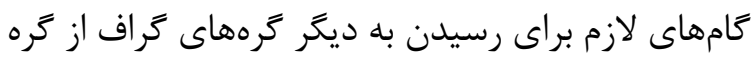

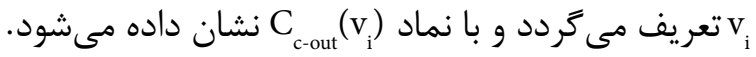

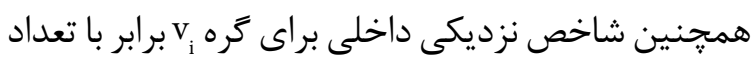

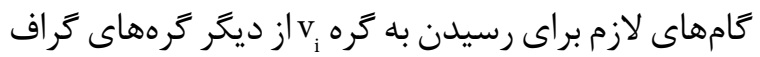

1 Walk

2 Path

3 Betweenness

4 Closeness

5 In-Closeness

6 Out-Closeness

Iran Occupational Health. 2021 (01 July);18: 14 
حداكثر مساحت حوضجه براى سناريوى حريق هر مخزن با توجه به مساحت باند وال مربوطه در نظر ترفته شدئ

مرحله سوم: تعيين برد/رهاى تشديد با توجه به سناريوى

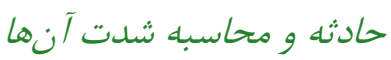
در اين مرحله بر اساس نوع حادثه در نظر گرفتتهشده

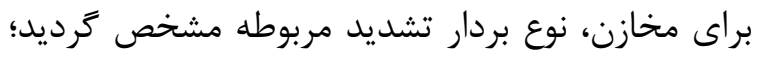

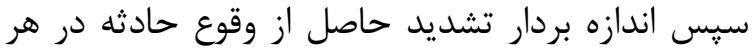

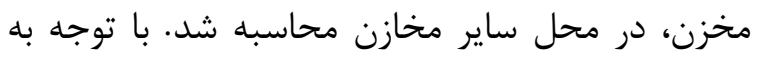

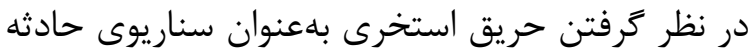

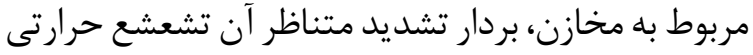

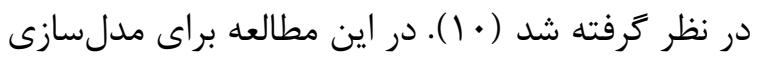

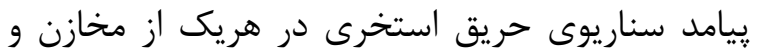

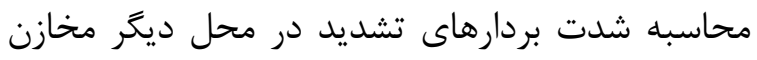

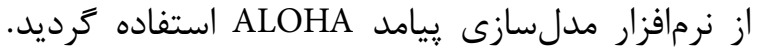

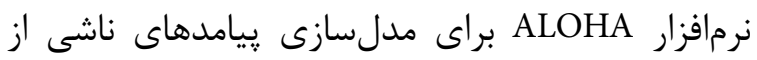

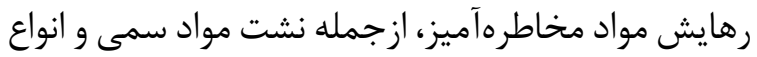

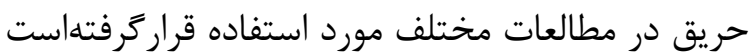

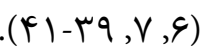

مرحله جِهارم: مقايسه بردارهاى تشديد با مقدار رآتانه و

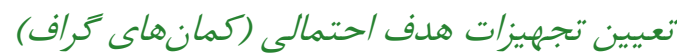

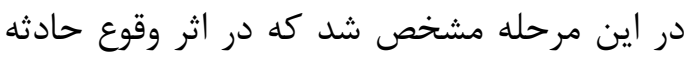

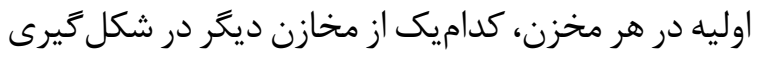

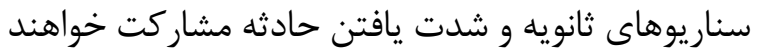

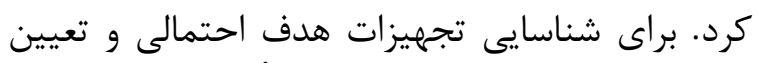

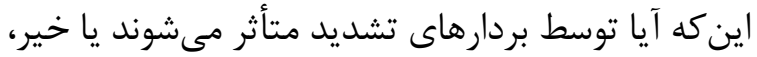

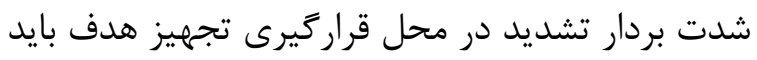

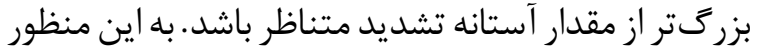

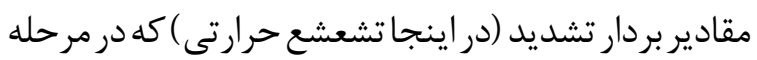

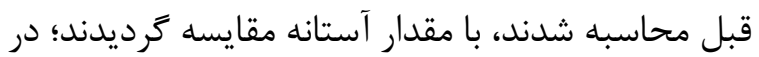

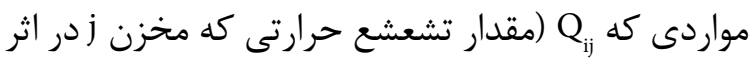

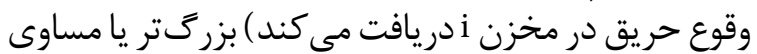

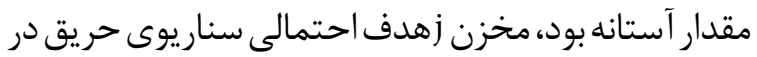

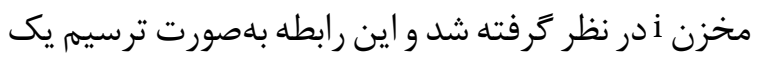

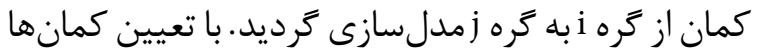

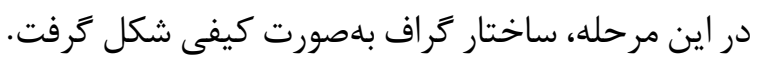

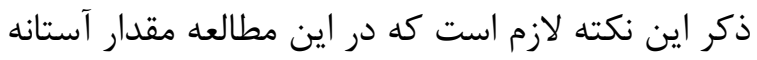

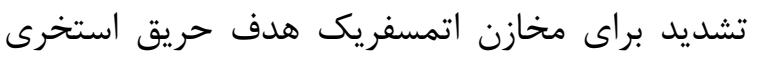

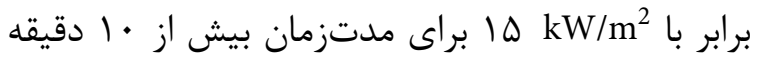

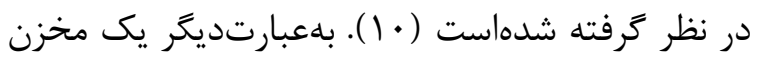

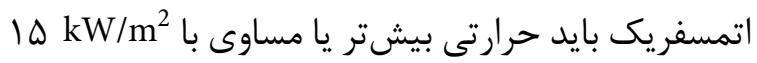

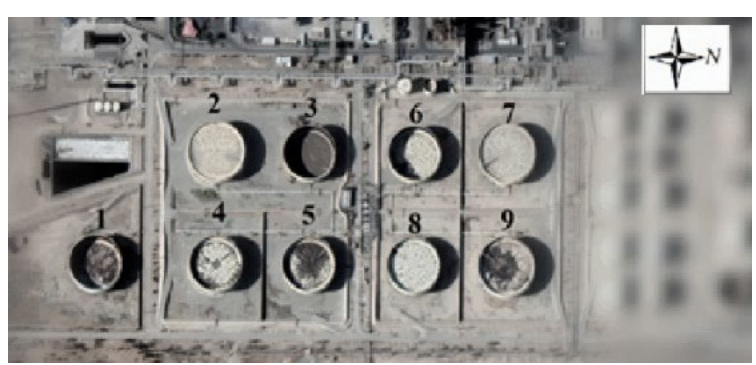

شكل ا. جانمايى مخازن تحت بررسى در مطالعه موردى.

از نوع اتمسفريك و داراى سقف شناور بوده و نسبت به

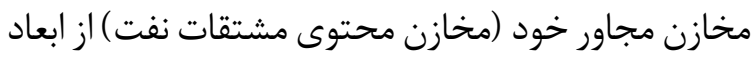

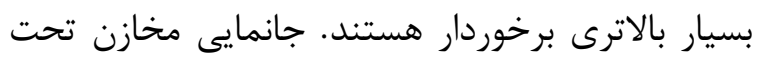

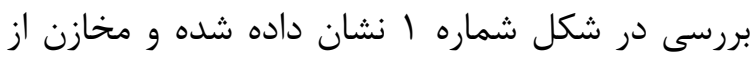

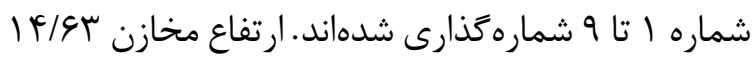

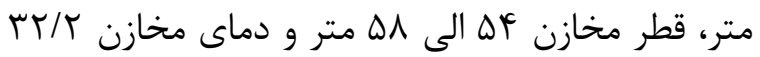

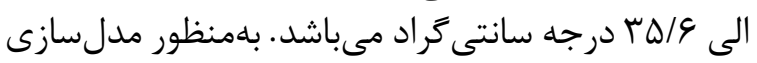

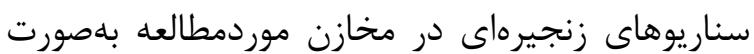

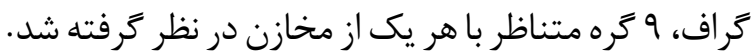

مرحله دوم: تعيين سناريوى حادثه براى هريك /ز مجان تجهيزات مخاطرةآميز

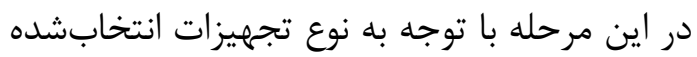

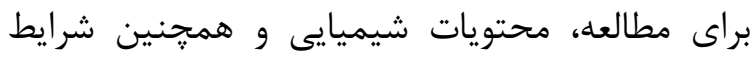

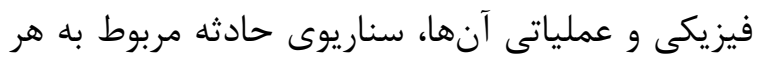

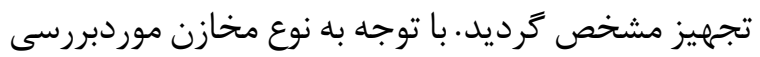

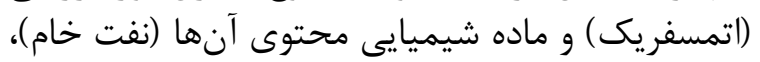

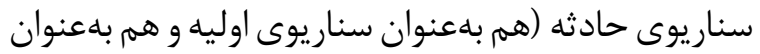

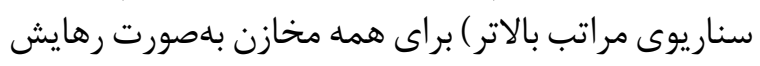

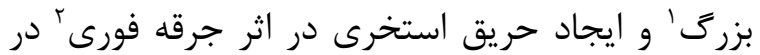

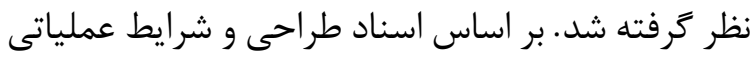

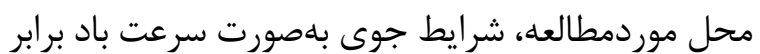

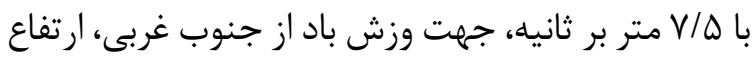

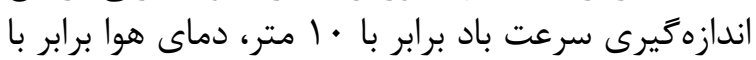

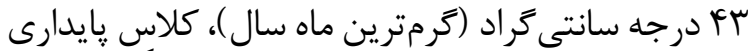

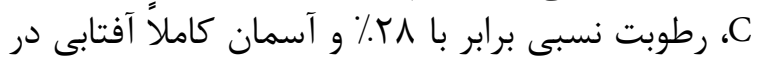

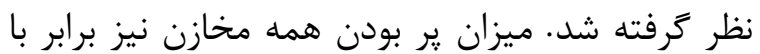

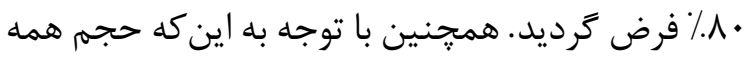

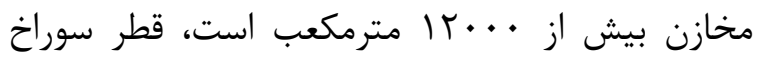

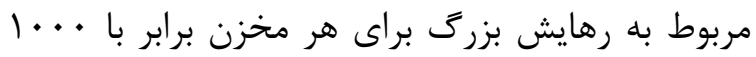

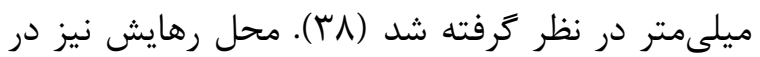
ارتفاع صفر از كف مخزن (بدترين سناريو) فرض كرضئ شرديد.

1 Major Release

2 Immediate Ignition 
مرحله هغتم: تحليل آسيبذيذيرى تجهيزات بر /ساس

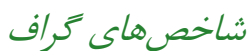

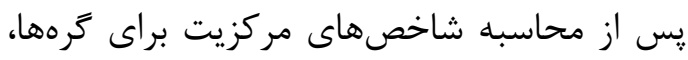

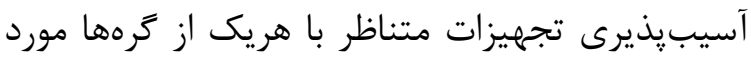

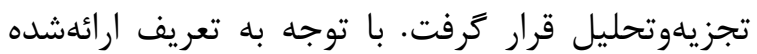

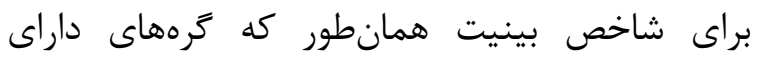
بينيت بالاتر نقش واسطه را در كراف براف ايفا مى كنند،

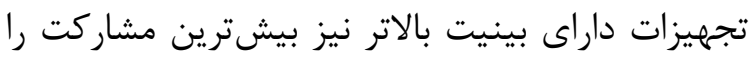

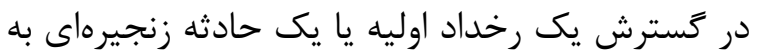

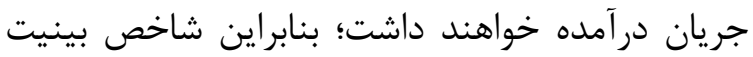

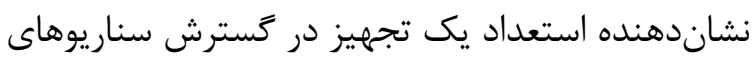

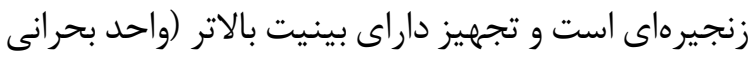

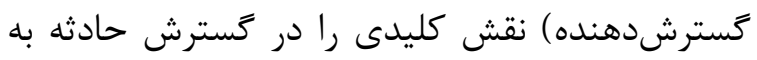

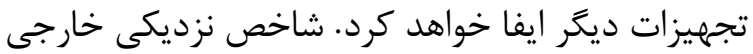

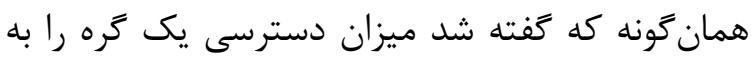

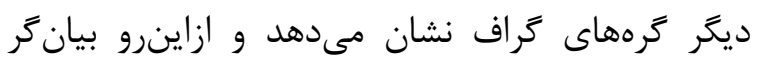

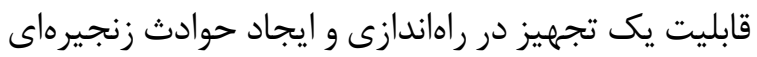

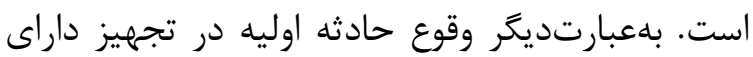

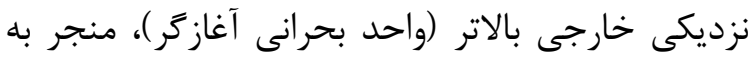

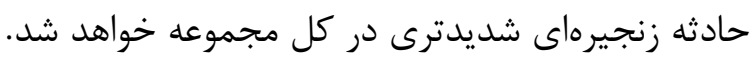

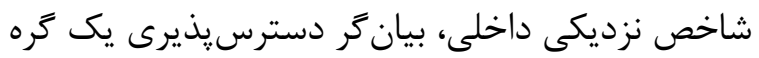

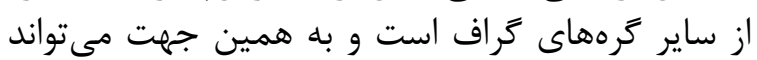
قابليت يك تجهيز را در تأثرافير إنذيرى حين هين سناريوهاى

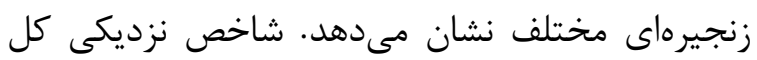

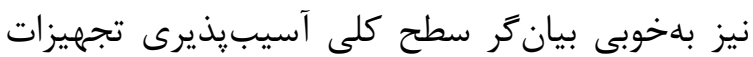

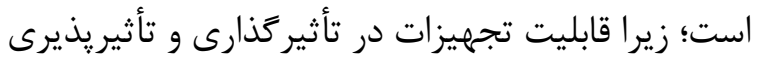

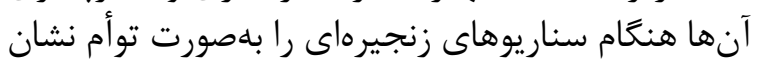

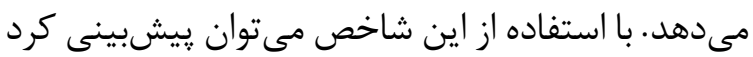

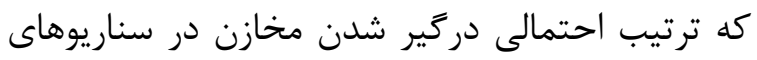

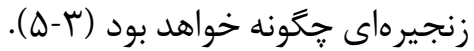

بافنته

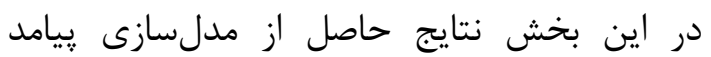

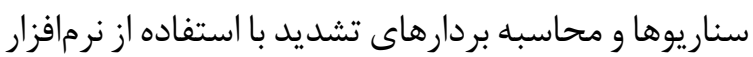

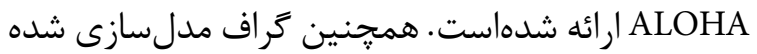

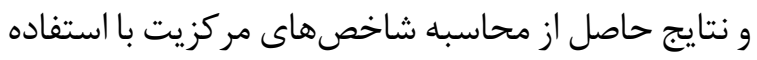

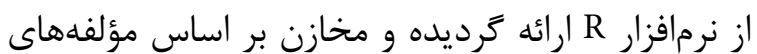
آسيب پيذيرى باهم مقايسه شدهاند.

نتايج حاصل /ز محاسبه بردارهاى تشديد و تعيين مخازن هدف از طريق مدلسازى سناريوى حريق تعريفشده در
را براى مدتزمان بيشتر از • ا دقيقه دريافت كند تا

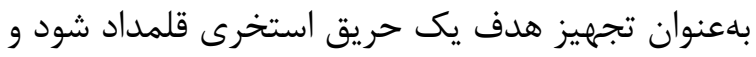
خود موجب شكل خيرى سناريوى مرتبه بالاتر گر دد. مرحله پينجم: محاسبه وزن كمانهاى تراف

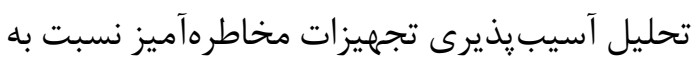

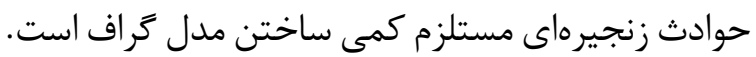

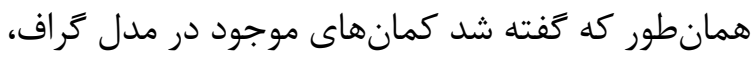

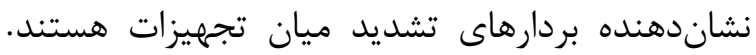

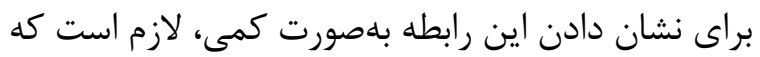

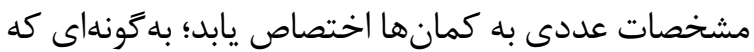

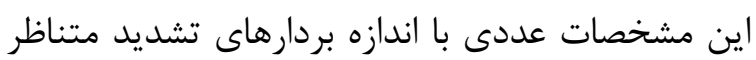

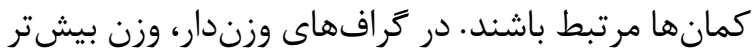

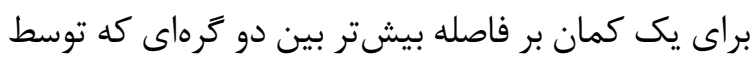

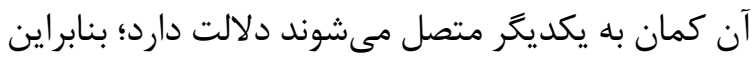

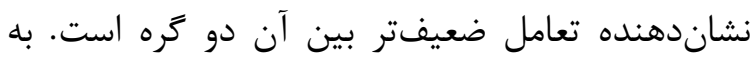

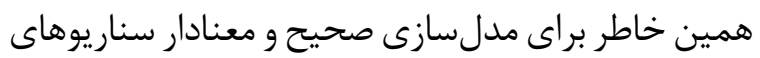

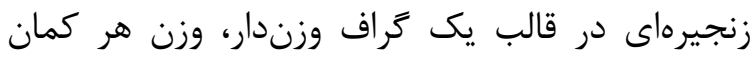

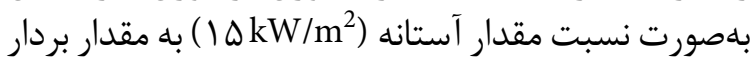

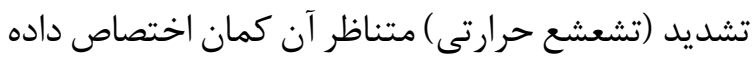

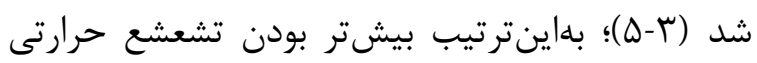

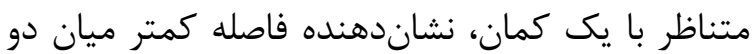

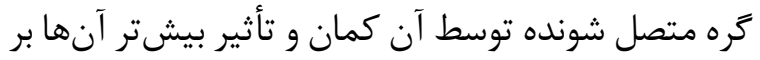
يكديكر در طول حادثه زنجيرهاى است.

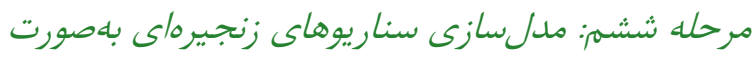

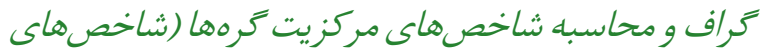
آسيب پِنيرى تجهيزات متناظر)

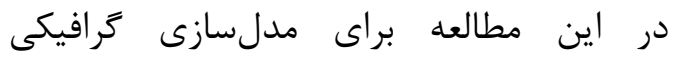

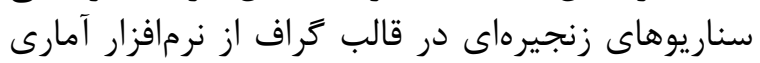

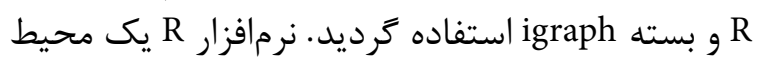
برنامهنويسى آمارى متنباز است كه در آن با باسته استفاده از

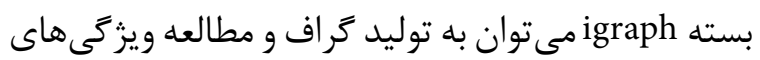

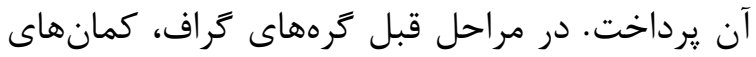

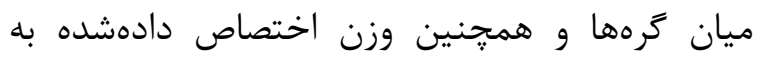

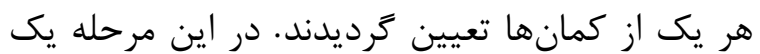

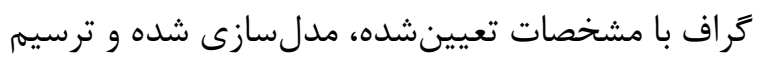

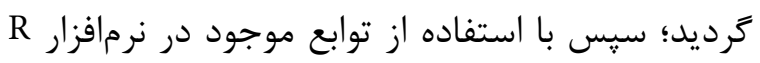

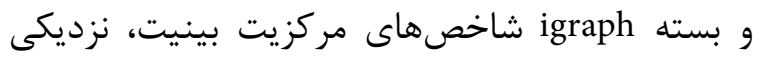

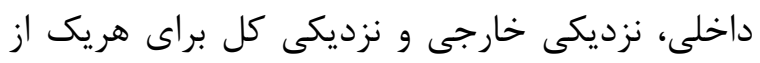

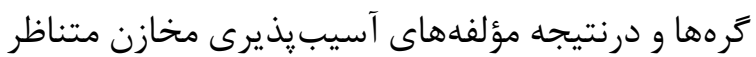
آنها محاسبه شدند. 
ᄉ مخزن ديخر نيز تعيينشده و مقدار تشعشع حرارتى

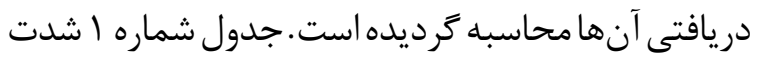

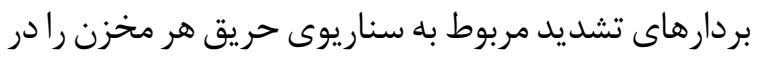

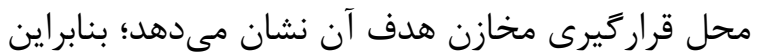

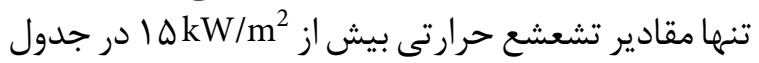

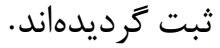

نتايج حاصل /ز مدلسازى كراف متناظر با سناريوهاى

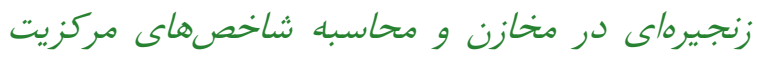

Loo

يس از تعيين مخازن هدف و كمان كمان كاى كراف، وزن

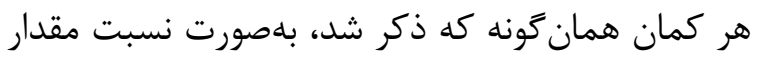

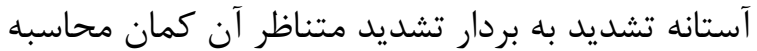

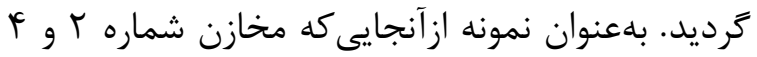

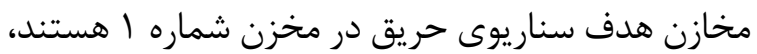

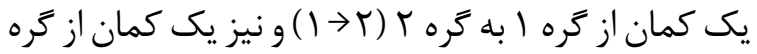

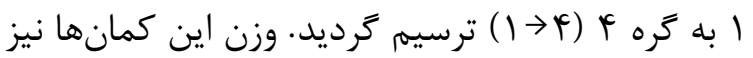

هر مخزن با استفاده از نرمافزار ALOHA، كليه بردارهاى

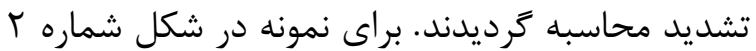

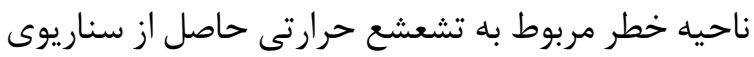
حريق در مخزن شماره ه با استفاده از نرمافزار

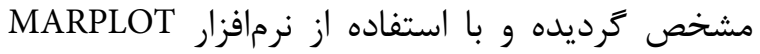

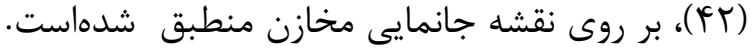

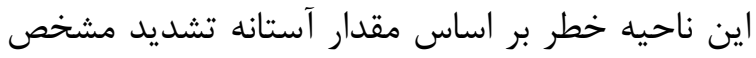

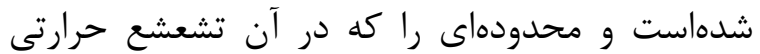

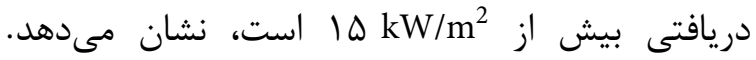

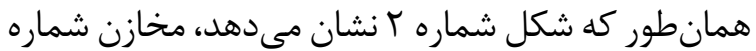

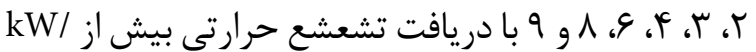

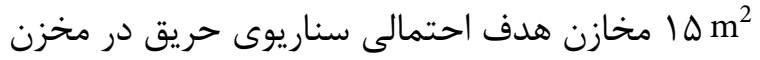

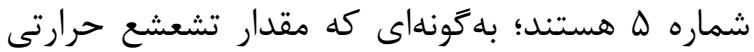

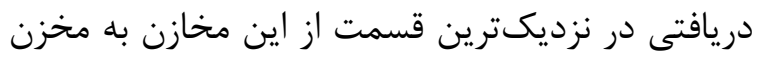

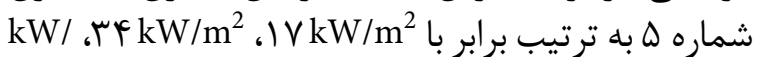

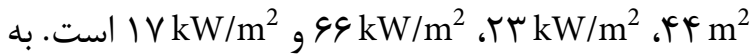
همين ترتيب مخازن هدف مربوط به سناريوى حريق در
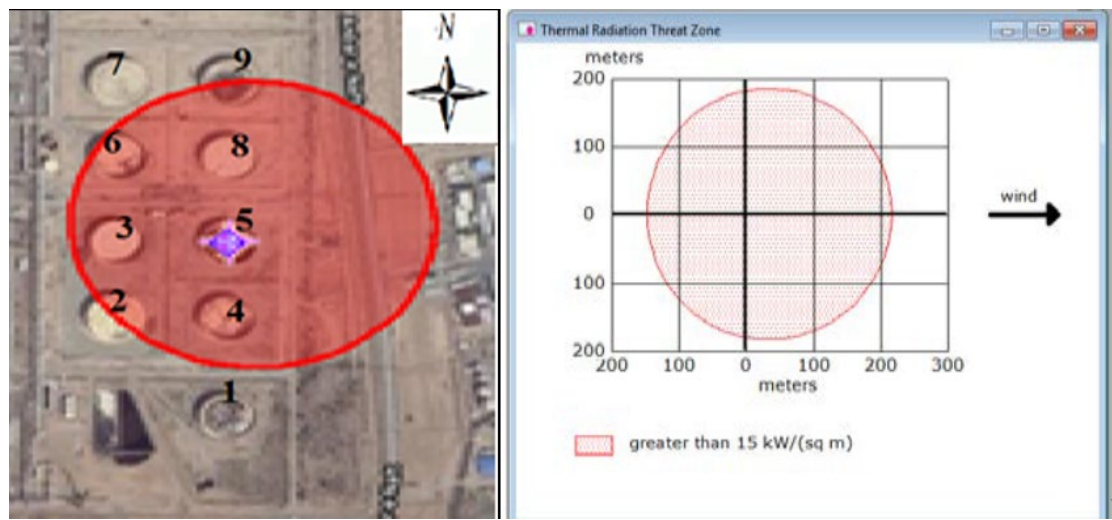

شكل ז. ناحيه خطر و مخازن هدف مربوط به حريق استخرى در مخزن شماره ه (بر اساس مقدار آستانه تشديد) كه با استفاده از نرمافزارهاى MARPLOT, ALOHA

جدول ا. مقادير تشعشع حرارتى (kW/m²) ناشى از وقوع حريق در مخزن i در محل قراركيرى مخزن هدف j.

\begin{tabular}{|c|c|c|c|c|c|c|c|c|c|}
\hline 9 & $\wedge$ & v & 4 & $\Delta$ & r & $r$ & $r$ & 1 & $\begin{array}{l}\mathrm{j} \\
\mathrm{i}\end{array}$ \\
\hline- & - & - & - & - & $\Delta \cdot$ & - & $r$. & - & 1 \\
\hline- & - & - & 19 & r. & q. & $\Lambda \Delta$ & - & $I V / \Delta$ & r \\
\hline- & rq & 19 & 99 & G. & $r \Delta$ & - & $i_{\Delta}$ & - & r \\
\hline- & 19 & - & - & $\Lambda \Delta$ & - & $r \Delta$ & $r F$ & $r \Delta$ & f \\
\hline IV & 99 & - & r & - & fr & $r F$ & IV & - & $\Delta$ \\
\hline FT & G. & $\Lambda \Lambda$ & - & r & - & ra & - & - & 9 \\
\hline 41 & ra & - & il & - & - & - & - & - & $v$ \\
\hline$\Lambda \Delta$ & - & ra & ro & ra & - & 10 & - & - & $\wedge$ \\
\hline - & Fr & ra & 19 & - & - & - & - & - & $q$ \\
\hline
\end{tabular}




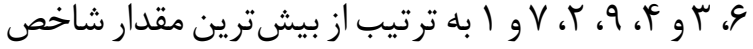

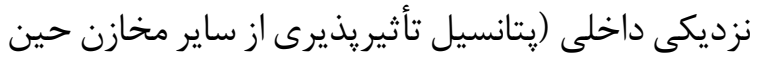

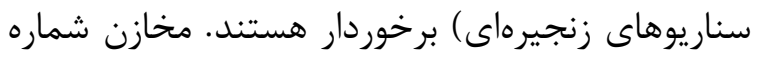

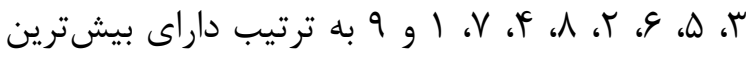

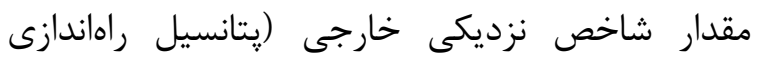

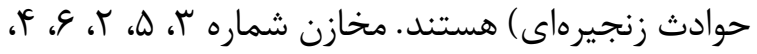

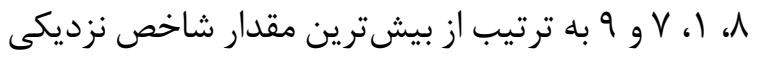

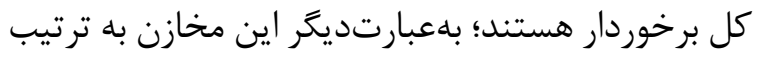

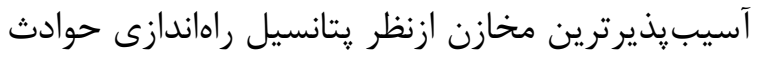

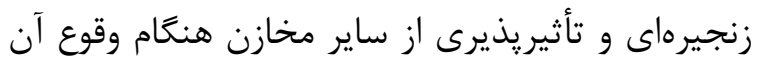
بلصورت توأم هستند.

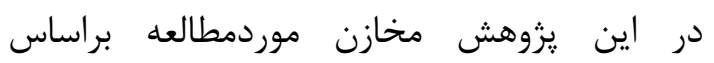

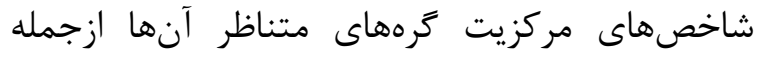

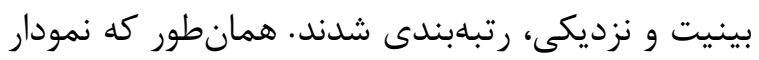

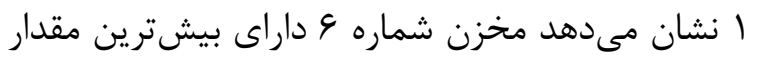

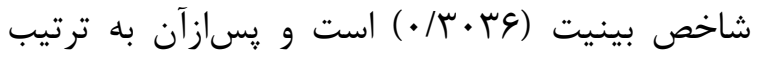

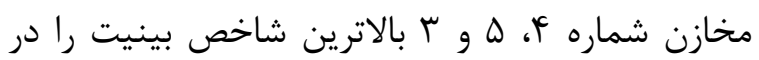

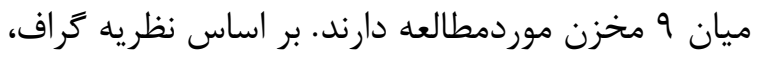

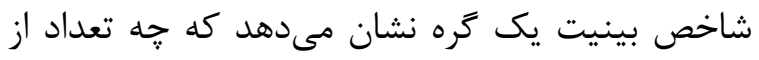

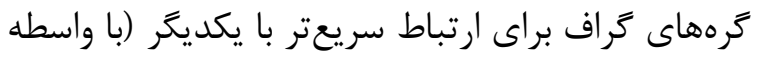

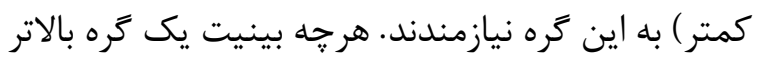

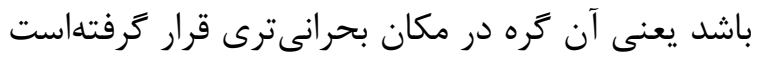

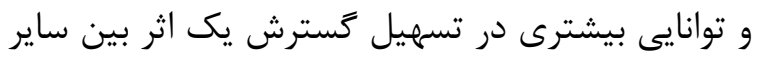

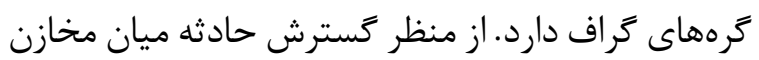

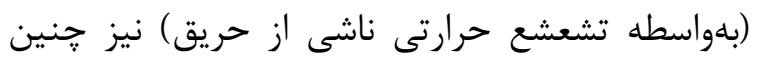

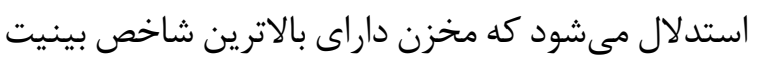

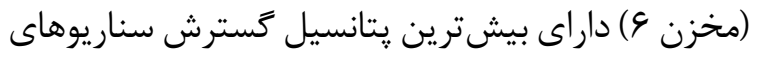

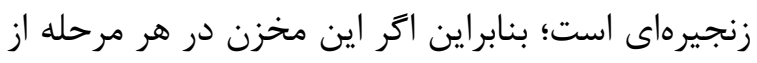

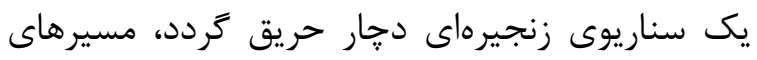

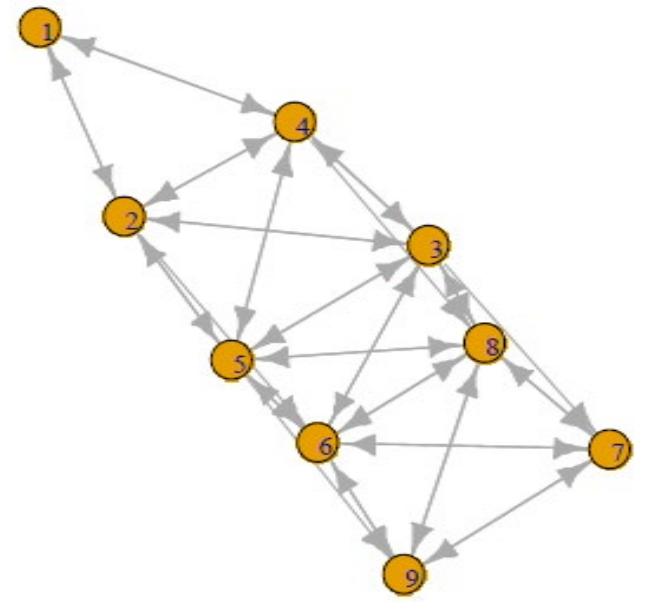

شكل r. مدلسازى كراف متناظر با سناريوهاى زنجيرهاى در مخازن

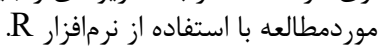

به ترتيب بلهورت (

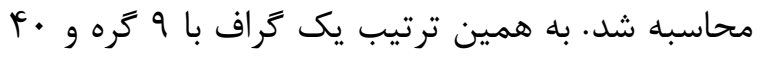

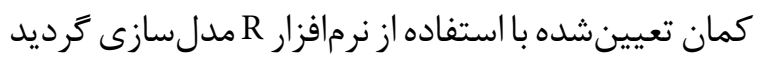

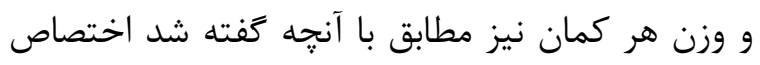

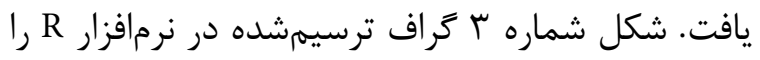

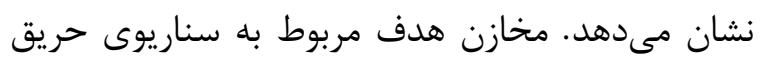

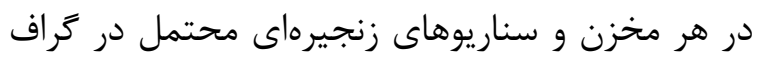

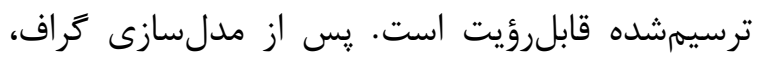

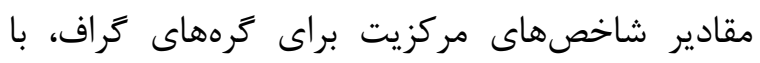

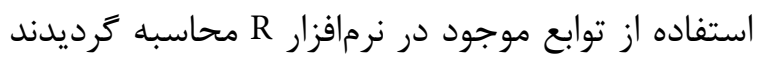

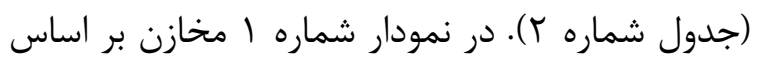

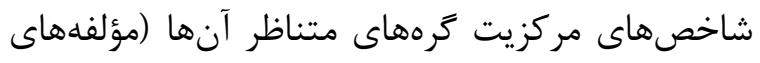

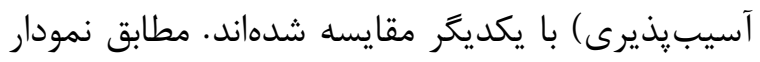

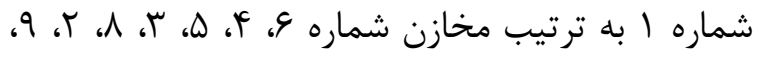

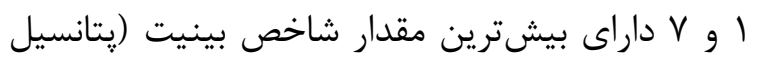

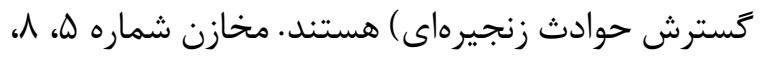

جدول r. شاخصهاى مركزيت محاسبهشده براى هريك از گرهها (مخازن).

\begin{tabular}{|c|c|c|c|c|}
\hline شاخص نزديكى كل & شاخص نزديكى خارجى & شاخص نزديكى داخلى & شاخص بينيت & شماره مخزن \\
\hline 1 & 1 & $\cdot / V \wedge \cdot \Delta$ & $\cdot$ & 1 \\
\hline I/FTER & 1/אזG & $1 / .9 F f$ & $\cdot / \cdot r \Delta V$ & r \\
\hline I/Vৎ9. & I/Vৎ६. & I/rqu & . & r \\
\hline I/TqTF & I/TGTF & 1/rq & $\cdot \pi \Delta$ & q \\
\hline $\mid / \Delta F I F$ & $|/ \Delta F| F$ & $1 / 91 \wedge \Delta$ & . & $\Delta$ \\
\hline I/TNIV & $1 / \Delta 1 \Lambda$ & $1 / 4 \Delta 91$ & ( & 4 \\
\hline$\cdot / 99 \wedge \Delta$ & $1 / .911$ & $1 / \cdot v \cdot 9$ & . & $v$ \\
\hline I/THTV & ( & I/GAVA & $\cdot / 19 \cdot V$ & $\wedge$ \\
\hline . $/ 1999$ &.$/ 9 \Delta \wedge 1$ & $1 / \pi / 10$ &.$/ .1 \vee \wedge$ & 9 \\
\hline
\end{tabular}




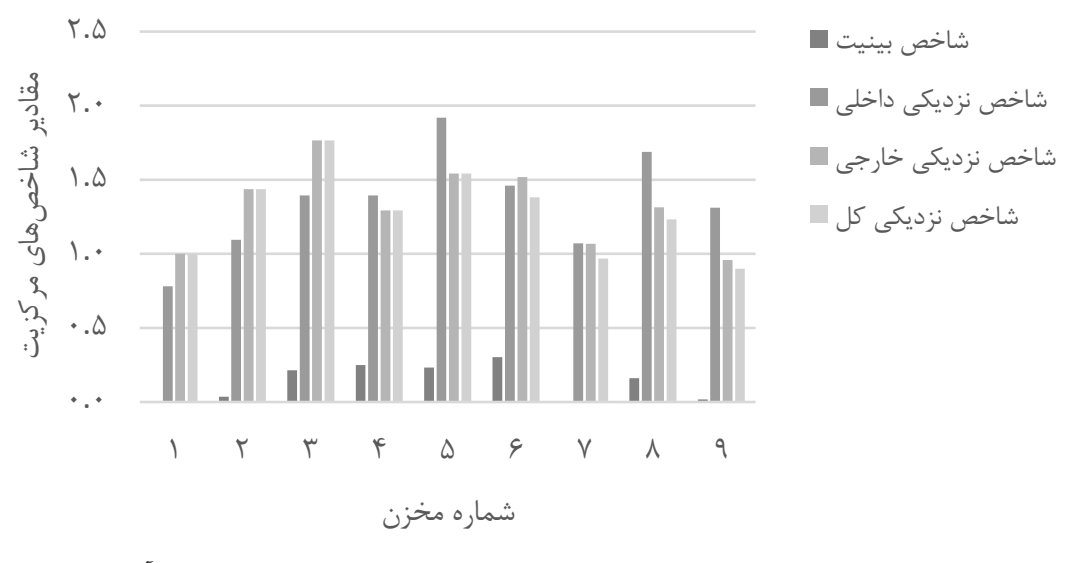

نمودار ا. مقايسه مخازن بر اساس مقادير شاخصهاى مركزيت گرههاى متناظر آنها.

مقدار نزديكى خارجى است، سرعت دسترسى بيشترى به

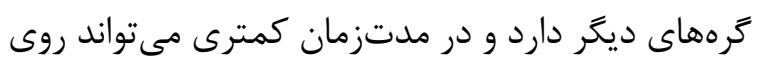

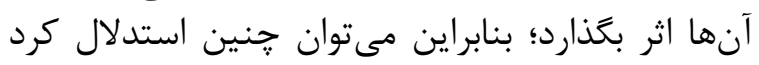

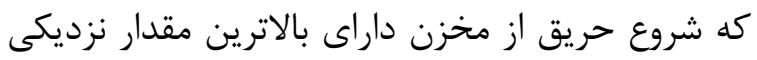

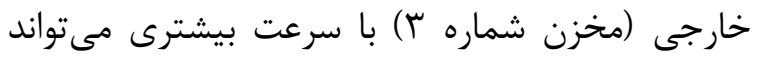

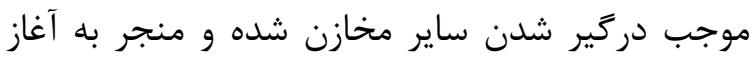

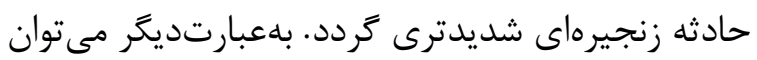

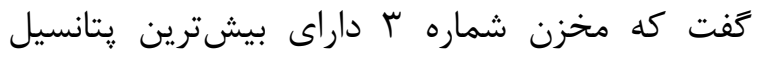

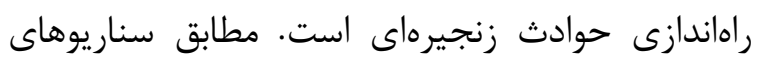

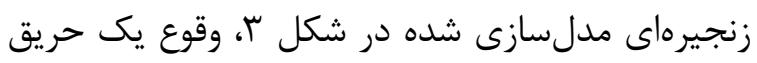

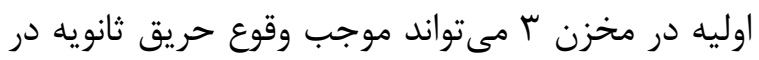

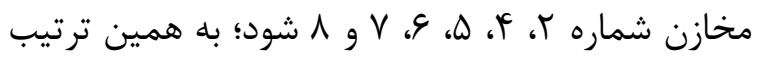

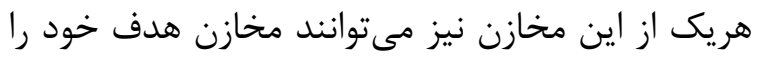

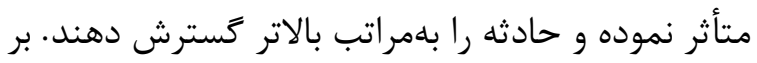

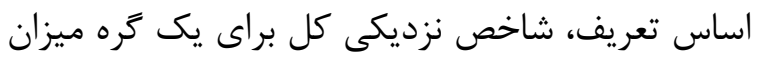

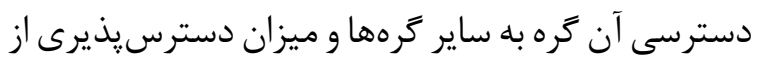

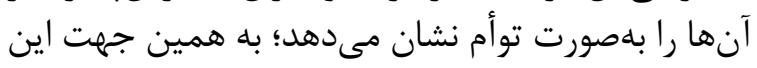

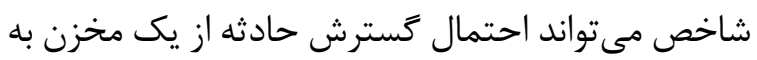

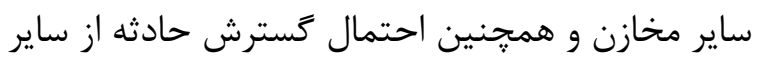

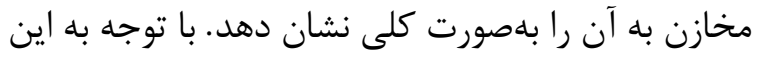

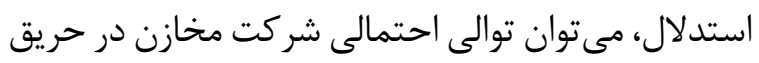

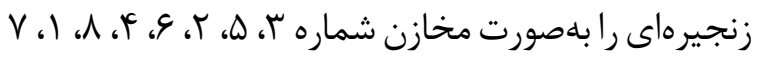

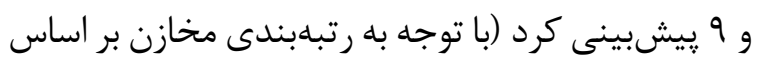
شاخص نزديكى كل در نمودار (1) ).

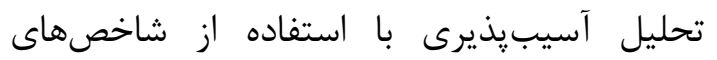

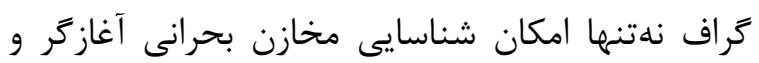

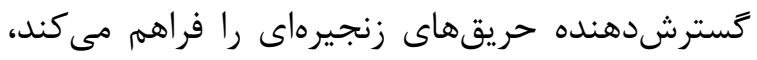

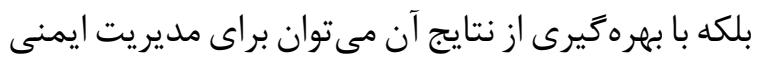

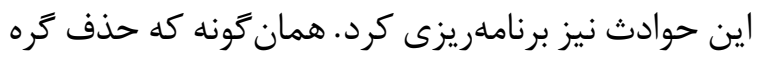

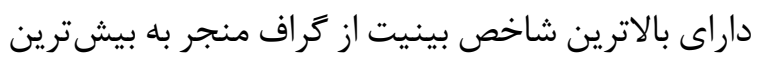

كسترش حادثه را تا بالاترين حد ممكن تسهيل خواهد

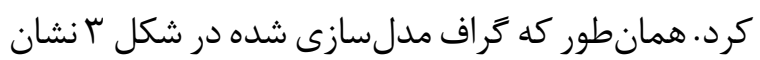

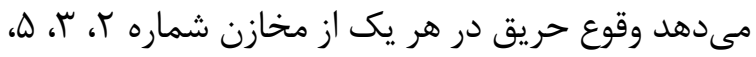

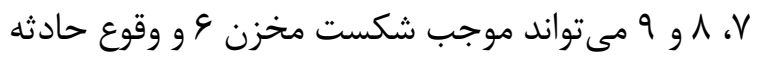

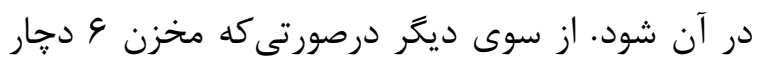

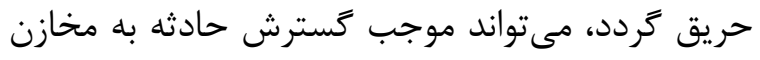

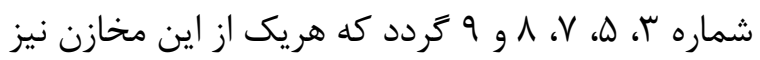

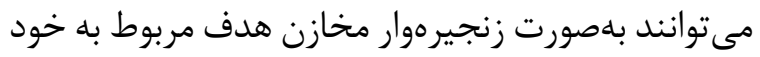

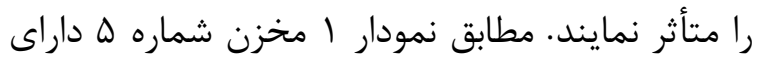

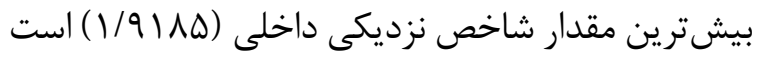

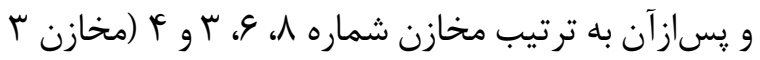

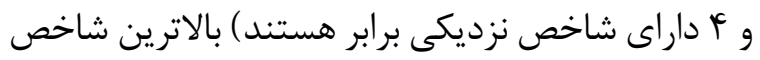

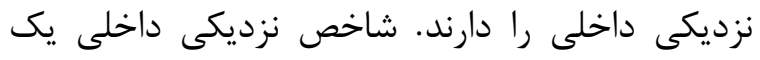

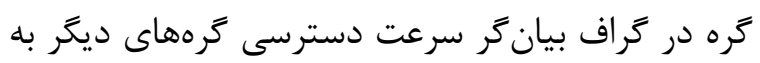

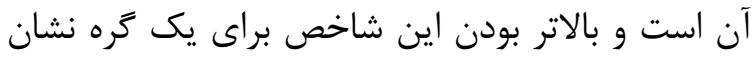

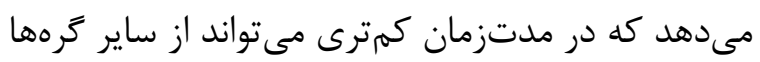

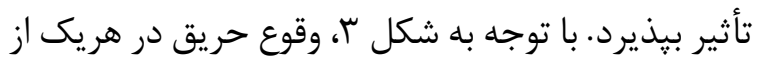

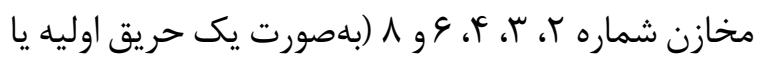

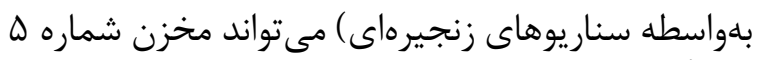

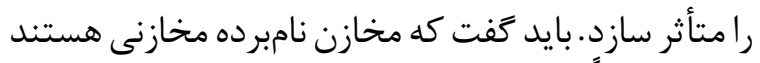

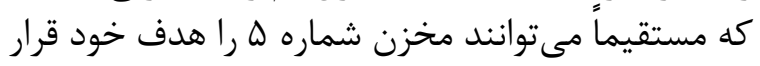

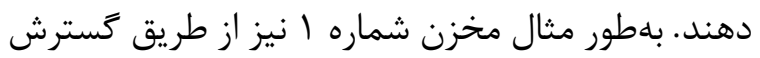

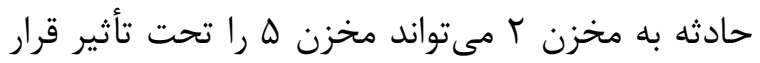

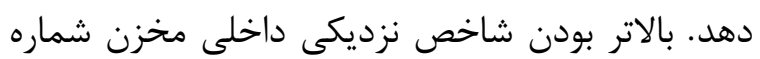

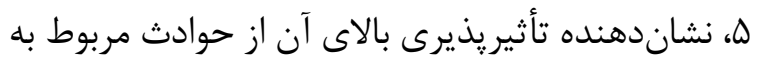

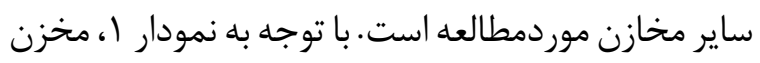

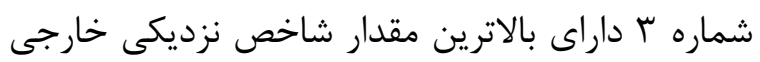

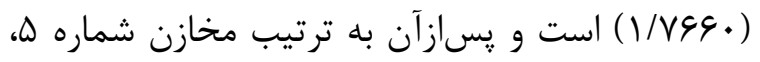

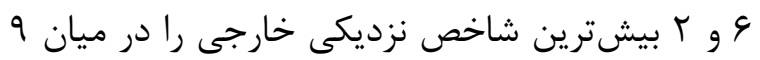

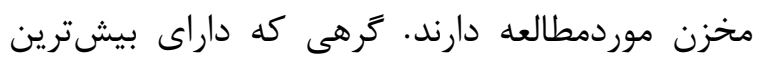


مجموعه مخازن مورد بررسى قرارگرفتهاست. نمودار

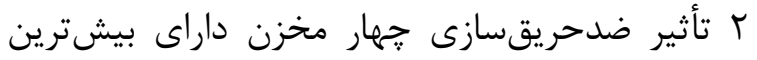

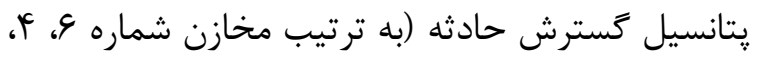

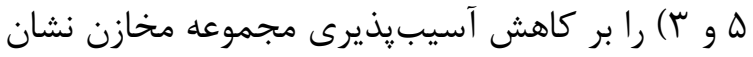

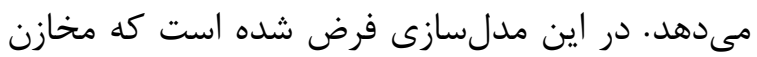

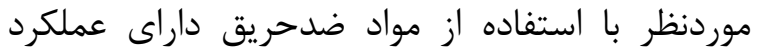

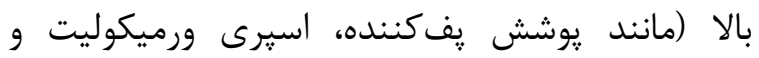

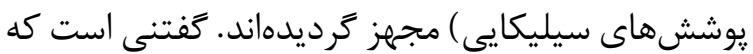

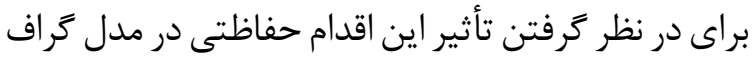

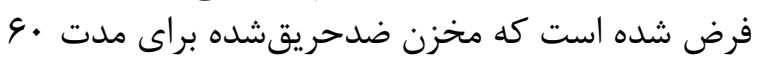

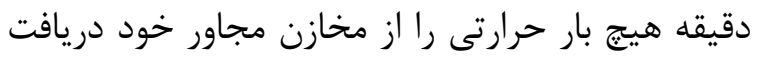

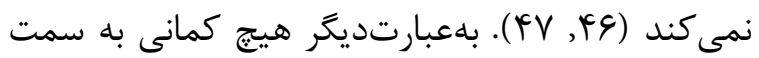

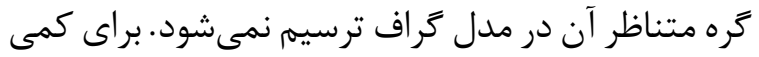

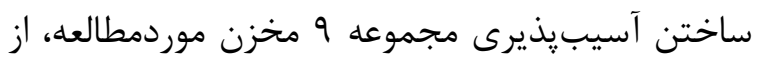

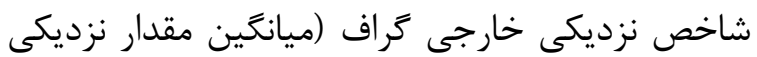

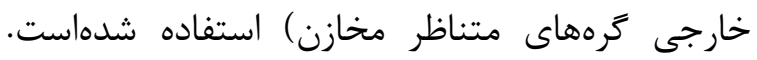

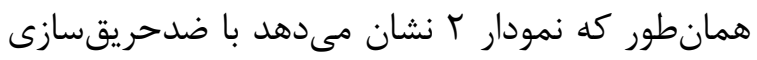

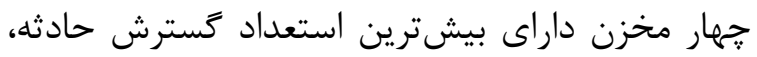

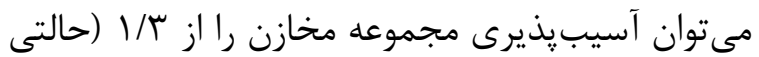

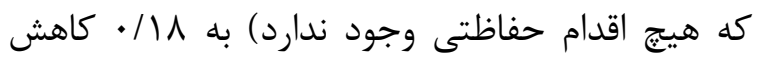

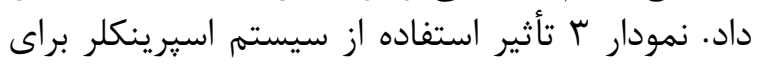

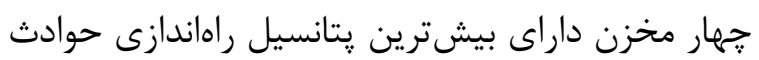

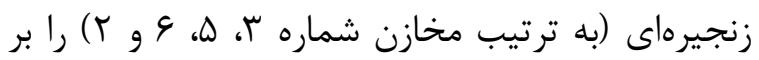

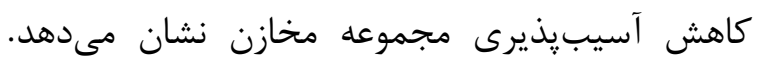
براى در نظر كرفتن تأثير اين اقدام حفاظتى درئ در مدل مدل

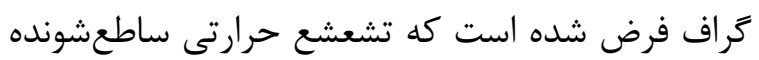

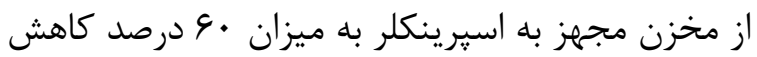

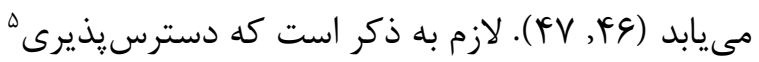

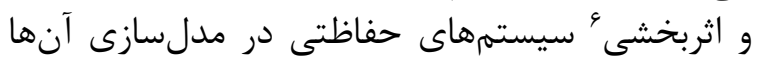

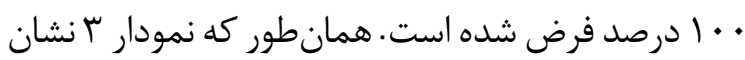

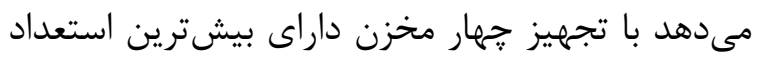

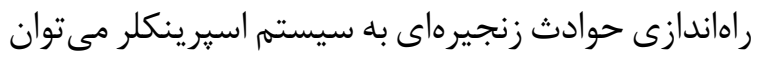

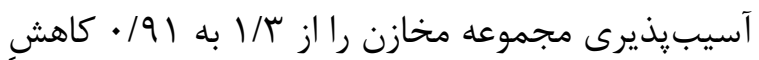

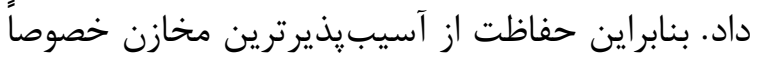

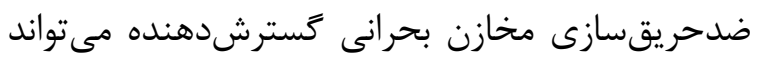

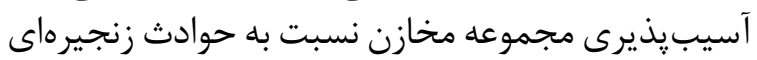

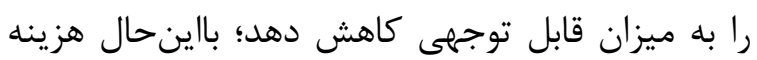

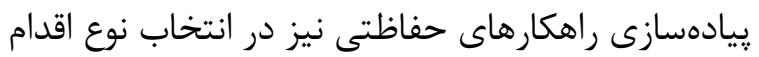

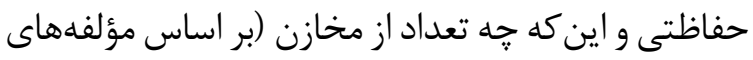

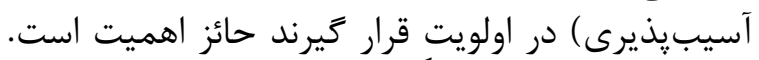

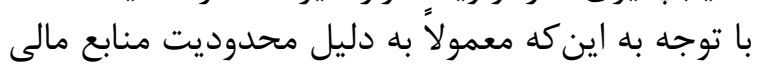

5 Availability 6 Effectiveness
گسستگى و انفصال در ساختار گراف مى گردد، محافظت

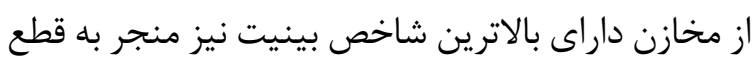

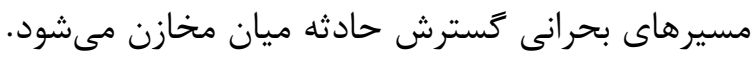

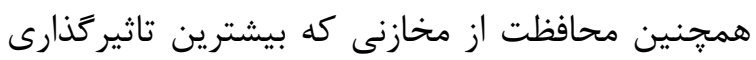

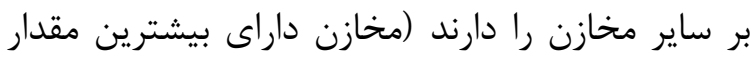

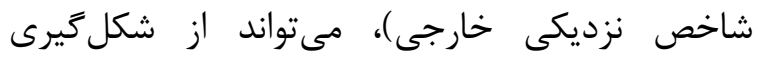

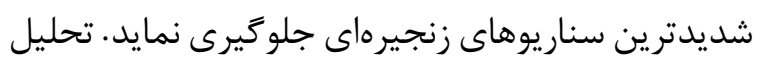

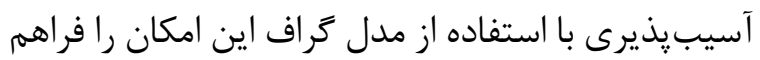

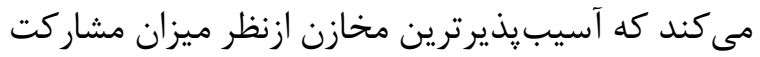

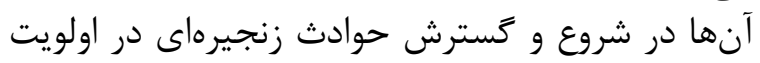

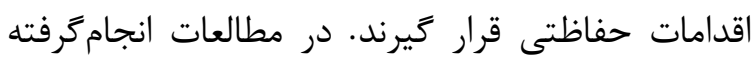

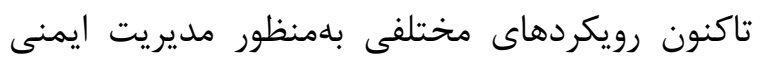

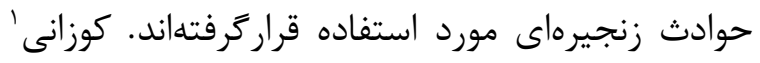

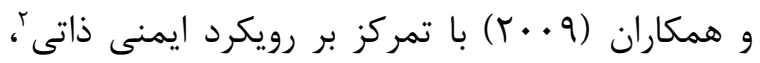

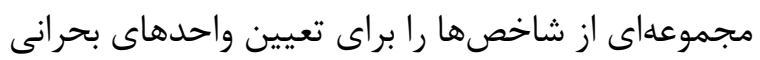

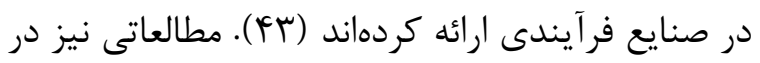

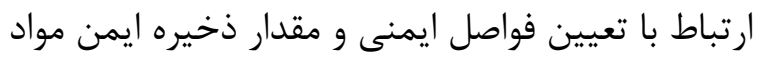

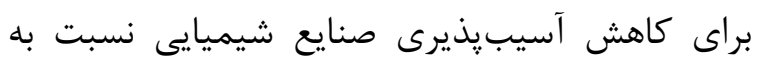

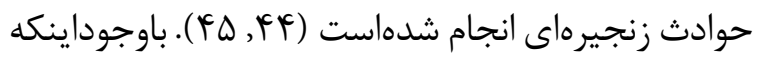
رويكردهاى ايمنى ذاتى مانى ماندام فراست فواصل ايمنى مئى ميان

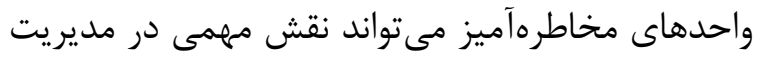

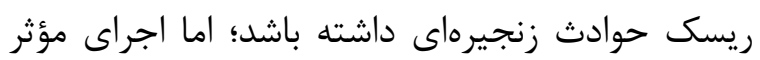

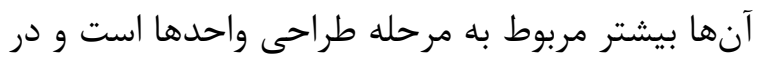

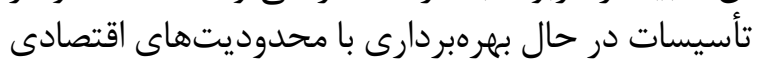

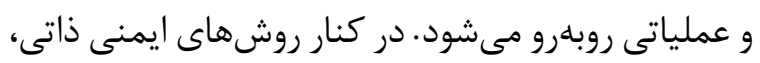

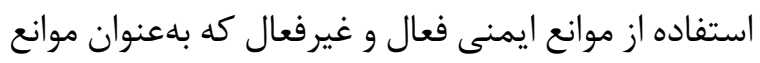

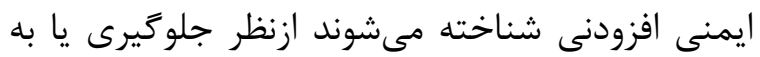

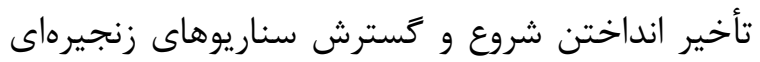

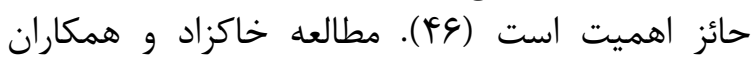
(Y • IV)

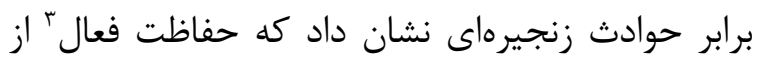

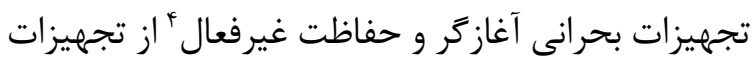

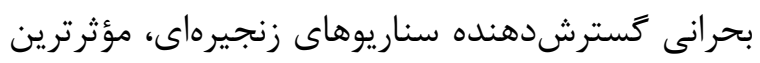

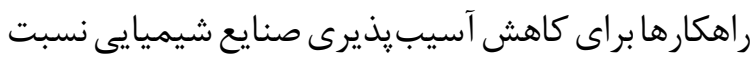

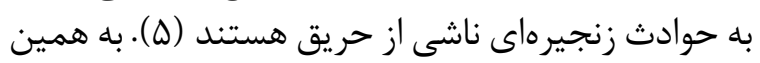

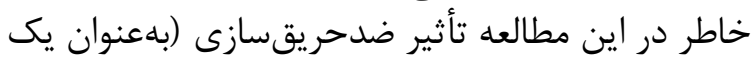

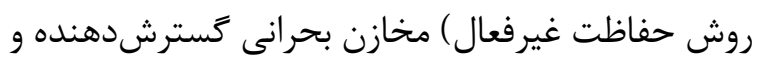

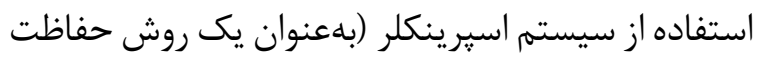

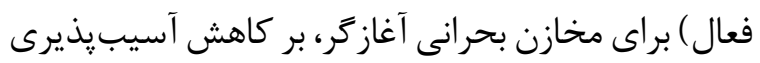

1 Cozzani

2 Inherent Safety

3 Active Protection

4 Passive Protection

Iran Occupational Health. 2021 (01 July);18: 14. 


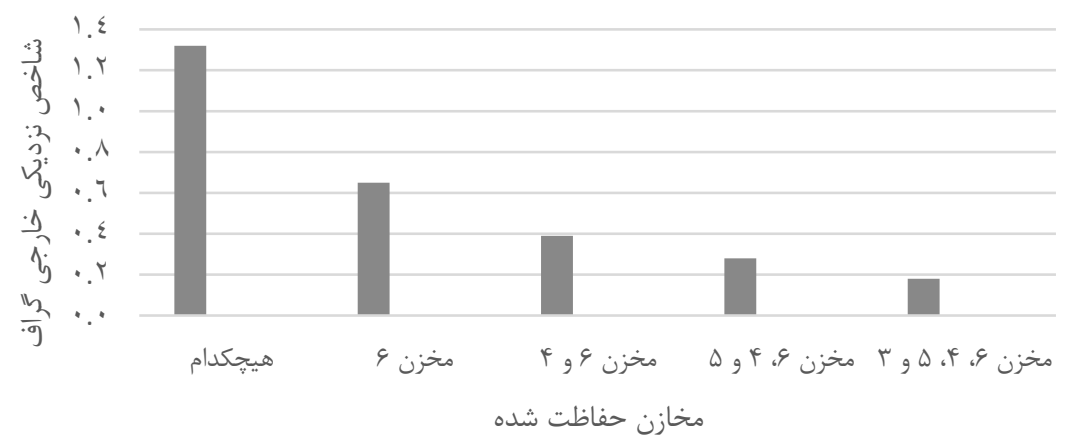

نمودار r. تأثير ضدحريقسازى مخازن بحرانى كسترشدهندهى سناريوهاى زنجيرهاى بر كاهش آسيبذيذيرى مجموعه مخازن.

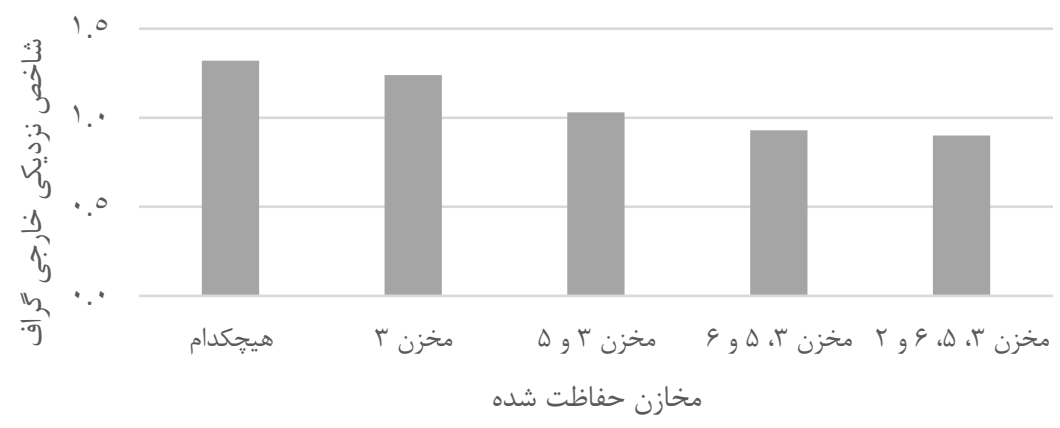

نمودار بّ. تأثير استفاده از سيستم اسيرينكلر براى مخازن بحرانى آغازَّر سناريوهاى زنجيرهاى بر كاهش آسيبذيذيرى مجموعه مخازن.

شايان ذكر است كه يكى از محدوديتهاى مطالعه

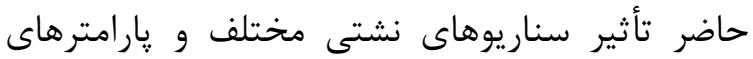

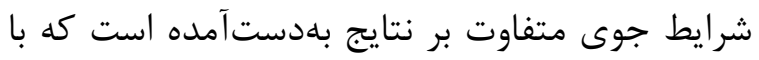

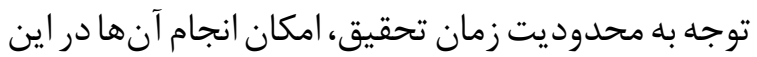

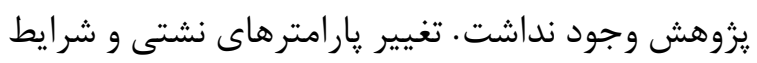

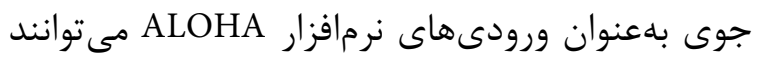
منجر به تغيير مقادير بردارهاى نشاي تشديد (تشعشع

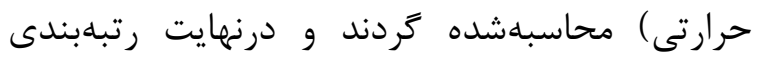

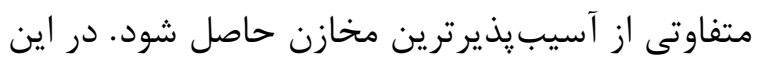

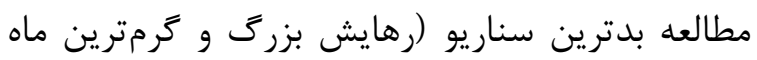

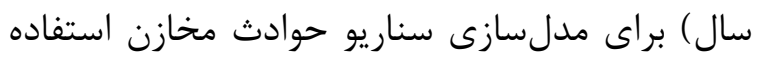

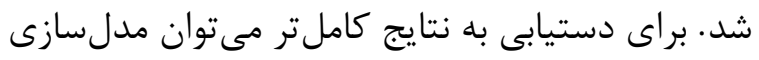

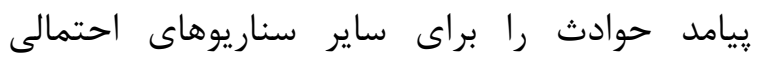

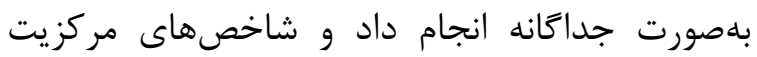

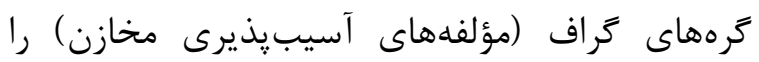

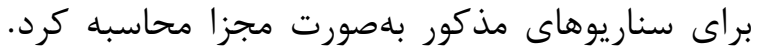

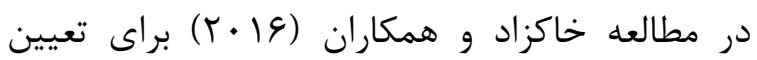

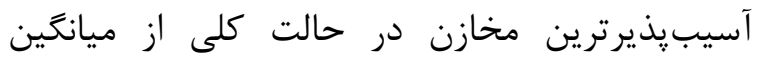

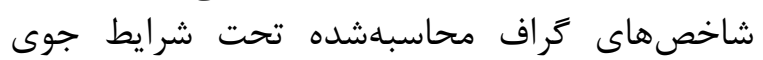

مختلف استفاده شدهاست (ץ) ماس (ب).
اقدامات حفاظتى نيازمند توجيه اقتصادى هستند، شاخص

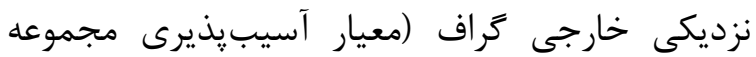

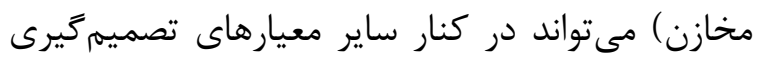

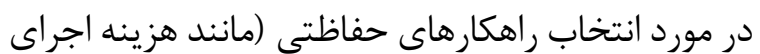

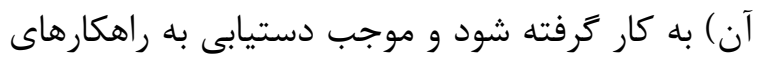
بهينه اقتصادى كردد. نتايج حاصل از تحليل آسيبذيذيرى بارديا استفاده از

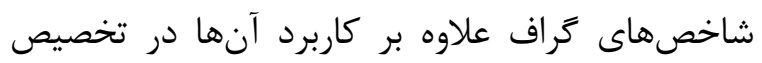

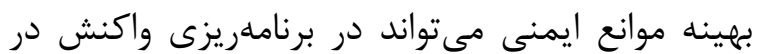

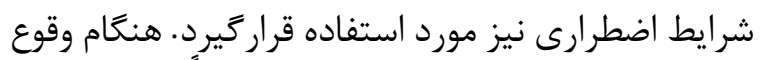

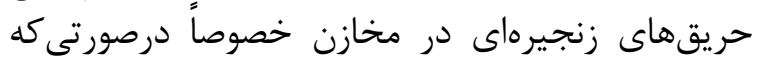

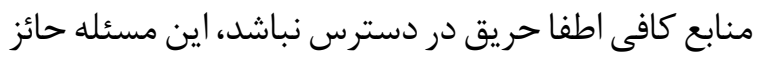

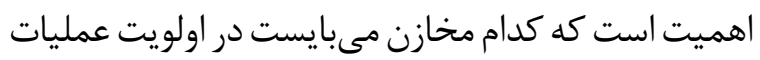

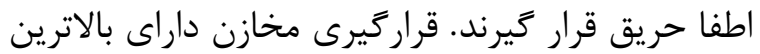

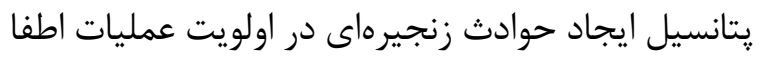

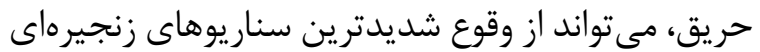

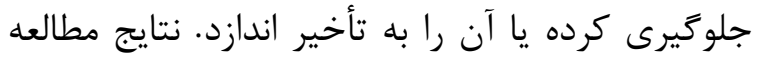

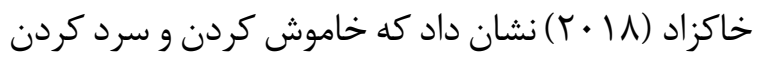

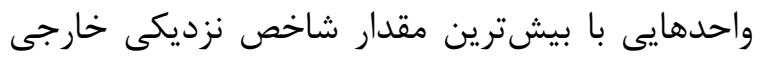
راهكار بهينه اطفا حريق است (YN) (F). 
made domino effects. Reliability Engineering \& System Safety. 2018.

3. Khakzad N, Reniers G, Abbassi R, Khan F. Vulnerability analysis of process plants subject to domino effects. Reliability Engineering \& System Safety. 2016;154:12736.

4. Khakzad N, Reniers G. Using graph theory to analyze the vulnerability of process plants in the context of cascading effects. Reliability Engineering \& System Safety. 2015;143:63-73.

5. Khakzad N, Landucci G, Reniers G. Application of Graph Theory to Cost-Effective Fire Protection of Chemical Plants During Domino Effects. Risk analysis. 2017;37(9):1652-67.

6. Jabbari M, Kavousi A. Consequence Analysis of Flammable Chemical Releases from a pipeline. The Fourth International Conference on Computational Sciences and Optimization (CSO 2011). Kunming and Lijiang, Yunnan, China, April 15-19, 2011.

7. Jabbari M, Atabi F, Ghorbani R. Key airborne concentrations of chemicals for emergency response planning in HAZMAT road transportation-margin of safety or survival. Journal of Loss Prevention in the Process Industries. 2020:104139.

8. Reniers G, Cozzani V. 3 - Features of Escalation Scenarios. Domino Effects in the Process Industries. Amsterdam: Elsevier; 2013. p. 30-42.

9. Necci A, Cozzani V, Spadoni G, Khan F. Assessment of domino effect: State of the art and research Needs. Reliability Engineering \& System Safety. 2015;143:3-18.

10. Cozzani V, Gubinelli G, Antonioni G, Spadoni G, Zanelli $S$. The assessment of risk caused by domino effect in quantitative area risk analysis. Journal of hazardous Materials. 2005;127(1):14-30.

11. Cozzani V, Gubinelli G, Salzano E. Escalation thresholds in the assessment of domino accidental events. Journal of hazardous materials. 2006;129(1):1-21.

12. Khakzad N, Reniers G. Application of Bayesian network and multi-criteria decision analysis to risk-based design of chemical plants. Chemical Engineering Transactions. 2016;48:223-8

13. Abdolhamidzadeh B. Present a model for evaluation of domino effects second level in process industrials [Thesis, Phd]. Tehran: Sharif University of Technology; 2010. [Persian].

14. Rad AH. Quantitative Risk Assessment of Domino Accidents using GIS [Thesis, M.Sc]. Tehran: Sharif University of Technology; 2011. [Persian].

15. Darbra R, Palacios A, Casal J. Domino effect in chemical accidents: main features and accident sequences. Journal of hazardous materials. 2010;183(1):565-73.

16. Abdolhamidzadeh B, Abbasi T, Rashtchian D, Abbasi
نتيجهاتَيرى

مدل نظريه گراف ابزار سودمندى براى مدلىسازى

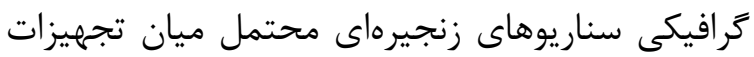

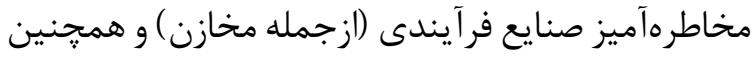

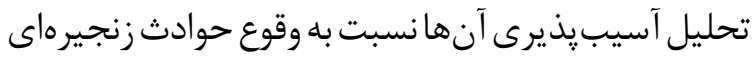

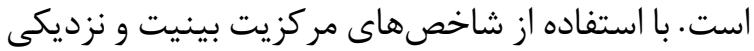

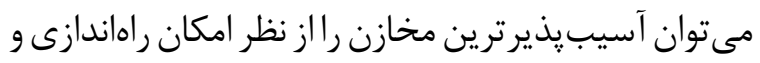

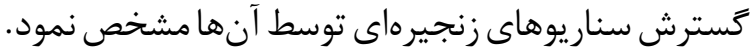

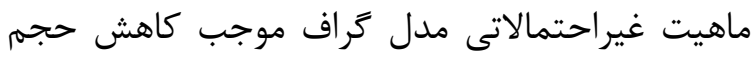

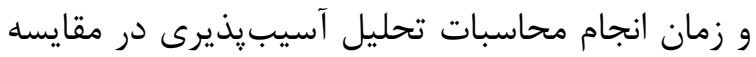
با روشهاى احتمالاتى مى گردد. نتايج حاصل إسبات از تحليل

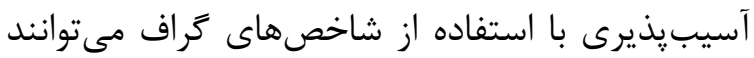

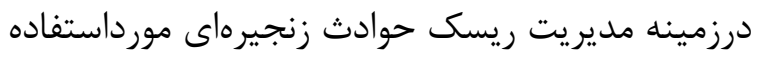

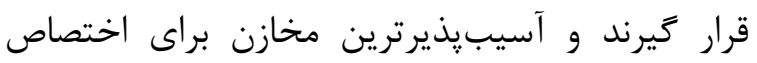

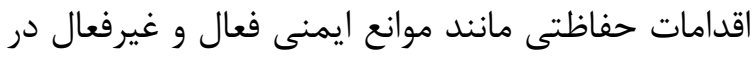

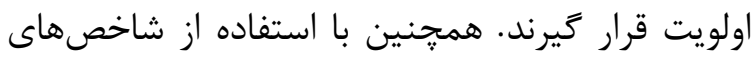
مركزيت مى توان ميزان كاهش آسيب

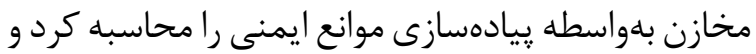

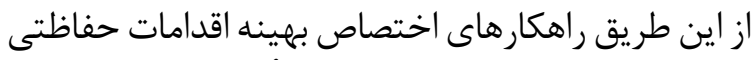

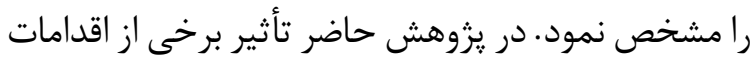

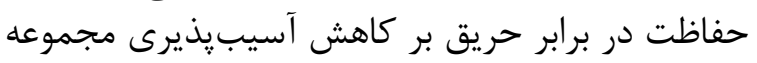

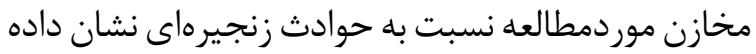

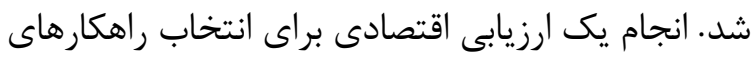

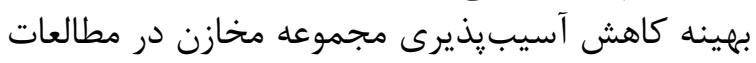

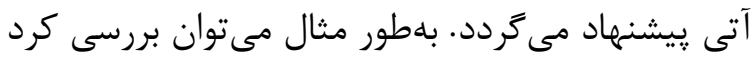

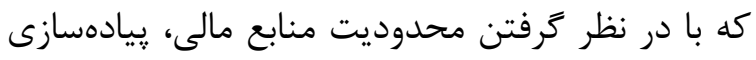

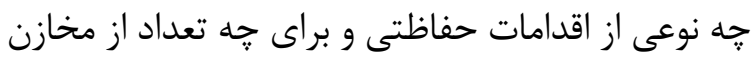

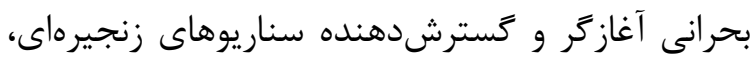

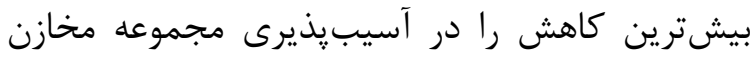
ايجاد خواهد كرد.

$$
\text { تشكر و قضردانى }
$$

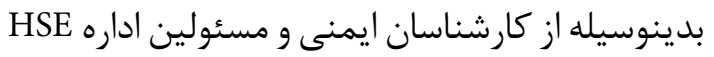
شركت يالايش نفت بابت همكارى ايشان در اجراى اين اين تحقيق تشكر و قدردانى مىنمايم.

منابع

1. Reniers GL, Audenaert A. Preparing for major terrorist attacks against chemical clusters: Intelligently planning protection measures wrt domino effects. Process Safety and Environmental Protection. 2014;92(6):583-9.

2. Khakzad N, Reniers G. Low-capacity utilization of process plants: A cost-robust approach to tackle man- 
facilities. Industrial \& Engineering Chemistry Research. 2018;57(11):3990-4006.

30. Chen C, Reniers G, Khakzad N. A thorough classification and discussion of approaches for modeling and managing domino effects in the process industries. Safety science. 2020;125:104618.

31. ALOHA. US Environmental Protection Agency, National Oceanic and Atmospheric Administration. Available online at: https://www.epa.gov/cameo/aloha-software.

32. R Development Core Team. R: A Language and Environment for Statistical Computing. Vienna, Austria: R Foundation for Statistical Computing, 2009.

33. Csardi G, Nepusz T. The igraph software package for complex network research. InterJournal, Complex Systems. 2006;1695(5):1-9.

34. Freeman LC. Centrality in social networks conceptual clarification. Social networks. 1978;1(3):215-39.

35. Sadeghi A, Jabbari Gharabagh m, Rezaeian M, Alidoosti A. Fire and Explosion Risk Assessment in a Combined Cycle Power Plant. Iranian Journal of Chemistry and Chemical Engineering (IJCCE). 2020.

36. Asghari B, Omidvari M. Probability assessment of chemical liquid release at floating roof storage tank in the oil refinery by fuzzy fault tree analysis. Iran Occupational Health. 2018;15(4):8-20. [Persian].

37. Chang JI, Lin C-C. A study of storage tank accidents. Journal of loss prevention in the process industries. 2006;19(1):51-9.

38. FRED, Failure Rate and Event Data for use within Risk Assessments, HSE 2012.

39. Atabi F, Ghorbani R, Jabbari M. Assessment of safe distance for five toxic materials commonly in the accidents of chemical road transportation using ALOHA and PHAST software and CEI index (Case Study: Tehran-Qazvin Highway). Iran Occupational Health. 2017;14(4):42-35. [Persian].

40. Jabbari M, Sajjadi S, Hossein M, Gholamnia R, editors. Determination of Airborne Quantity and Consequence Analysis of 1, 3-Butadiene Release from a Petrochemical Plant Pipeline. Applied Mechanics and Materials; 2013: Trans Tech Publ.

41. Beheshti MH, Hajizadeh R, Mehri A, Borhani Jebeli M. Modeling the result of hexane leakage from storage tanks and planning a emergency response programm in a petrochemical complex. Iran Occupational Health. 2016;13(1):69-79. [Persian].

42. MARPLOT. US Environmental Protection Agency, National Oceanic and Atmospheric Administration. Available online at: https://www.epa.gov/cameo/ marplot-software.

43. Cozzani V, Tugnoli A, Salzano E. The development of an inherent safety approach to the prevention of
SA. Domino effect in process-industry accidents-An inventory of past events and identification of some patterns. Journal of Loss Prevention in the Process Industries. 2011;24(5):575-93.

17. Cozzani V, Salzano E. The quantitative assessment of domino effects caused by overpressure: Part I. Probit models. Journal of Hazardous Materials. 2004;107(3):6780 .

18. Cozzani V, Antonioni G, Spadoni G. Quantitative assessment of domino scenarios by a GIS-based software tool. Journal of Loss Prevention in the process industries. 2006;19(5):463-77.

19. Antonioni G, Spadoni G, Cozzani V. Application of domino effect quantitative risk assessment to an extended industrial area. Journal of Loss Prevention in the Process Industries. 2009;22(5):614-24.

20. Zhou J, Reniers G. A matrix-based modeling and analysis approach for fire-induced domino effects. Process Safety and Environmental Protection. 2018;116:347-53.

21. Rad A, Abdolhamidzadeh B, Abbasi T, Rashtchian D. FREEDOM II: An improved methodology to assess domino effect frequency using simulation techniques. Process Safety and Environmental Protection. 2014;92(6):714-22.

22. Abdolhamidzadeh B, Abbasi T, Rashtchian D, Abbasi SA. A new method for assessing domino effect in chemical process industry. Journal of hazardous materials. 2010;182(1):416-26.

23. Zhang L, Landucci G, Reniers G, Khakzad N, Zhou J. DAMS: A Model to Assess Domino Effects by Using Agent-Based Modeling and Simulation. Risk analysis. 2018;38(8):1585-600.

24. Reniers GL, Dullaert W. Knock-on accident prevention in a chemical cluster. Expert systems with applications. 2008;34(1):42-9.

25. Reniers GL, Dullaert W. DomPrevPlanning: user-friendly software for planning domino effects prevention. Safety Science. 2007;45(10):1060-81.

26. Khakzad N. Application of dynamic Bayesian network to risk analysis of domino effects in chemical infrastructures. Reliability Engineering \& System Safety. 2015;138:263-72.

27. Khakzad N, Khan F, Amyotte P, Cozzani V. Domino effect analysis using Bayesian networks. Risk Analysis. 2013;33(2):292-306.

28. Khakzad N, Amyotte P, Cozzani V, Reniers G, Pasman $\mathrm{H}$. How to address model uncertainty in the escalation of domino effects? Journal of Loss Prevention in the Process Industries. 2018;54:49-56.

29. Ji J, Tong Q, Khan F, Dadashzadeh M, Abbassi R. Riskbased domino effect analysis for fire and explosion accidents considering uncertainty in processing 
تحليل آسيبذيذيرى مخازن يكى از پالايشگاههاى نفت...

46. Khakzad N, Landucci G, Cozzani V, Reniers G, Pasman H. Cost-effective fire protection of chemical plants against domino effects. Reliability Engineering \& System Safety. 2018;169:412-21.

47. Landucci G, Argenti F, Spadoni G, Cozzani V. Domino effect frequency assessment: The role of safety barriers. Journal of Loss Prevention in the Process Industries. 2016;44:706-17.

48. Khakzad N. A graph theoretic approach to optimal firefighting in oil terminals. Energies. 2018;11(11):3101. domino accidents. Accident Analysis \& Prevention. 2009;41(6):1216-27.

44. Khakzad N, Khan F, Amyotte P, Cozzani V. Risk management of domino effects considering dynamic consequence analysis. Risk Analysis. 2014;34(6):112838 .

45. Cozzani V, Tugnoli A, Salzano E. Prevention of domino effect: from active and passive strategies to inherently safer design. Journal of hazardous materials. 2007;139(2):209-19. 